ARTICLE

\title{
Serine-linked PARP1 auto-modification controls PARP inhibitor response
}

Evgeniia Prokhorova (10 1,7, Florian Zobel ${ }^{1,7}$, Rebecca Smith (1) 2, Siham Zentout (1) 2, lan Gibbs-Seymour (1) 1,6, Kira Schützenhofer ${ }^{1}$, Alessandra Peters (1) ${ }^{1}$, Joséphine Groslambert', Valentina Zorzini ${ }^{1}$, Thomas Agnew ${ }^{1}$,

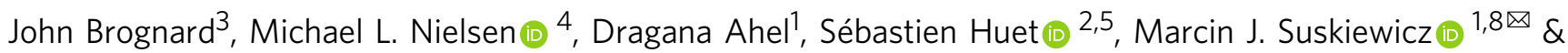
Ivan Ahel (1) 1,8凶

Poly(ADP-ribose) polymerase 1 (PARP1) and PARP2 are recruited and activated by DNA damage, resulting in ADP-ribosylation at numerous sites, both within PARP1 itself and in other proteins. Several PARP1 and PARP2 inhibitors are currently employed in the clinic or undergoing trials for treatment of various cancers. These drugs act primarily by trapping PARP1 on damaged chromatin, which can lead to cell death, especially in cells with DNA repair defects. Although PARP1 trapping is thought to be caused primarily by the catalytic inhibition of PARP-dependent modification, implying that ADP-ribosylation (ADPr) can counteract trapping, it is not known which exact sites are important for this process. Following recent findings that PARP1- or PARP2-mediated modification is predominantly serinelinked, we demonstrate here that serine ADPr plays a vital role in cellular responses to PARP1/PARP2 inhibitors. Specifically, we identify three serine residues within PARP1 (499, 507, and 519) as key sites whose efficient HPF1-dependent modification counters PARP1 trapping and contributes to inhibitor tolerance. Our data implicate genes that encode serinespecific ADPr regulators, HPF1 and ARH3, as potential PARP1/PARP2 inhibitor therapy biomarkers.

\footnotetext{
${ }^{1}$ Sir William Dunn School of Pathology, University of Oxford, Oxford, UK. ${ }^{2}$ Univ Rennes, CNRS, Structure Fédérative de Recherche Biosit, IGDR (Institut de Génétique et Développement de Rennes) - UMR 6290, Rennes, France. ${ }^{3}$ Laboratory of Cell and Developmental Signaling, Center for Cancer Research, National Cancer Institute, Frederick, MD, USA. ${ }^{4}$ Proteomics Program, Novo Nordisk Foundation Center for Protein Research, Faculty of Health and Medical Sciences, University of Copenhagen, Copenhagen, Denmark. ${ }^{5}$ Institut Universitaire de France, Paris, France. ${ }^{6}$ Present address: Department of Biochemistry, University of Oxford, Oxford, UK. ${ }^{7}$ These authors contributed equally: Evgeniia Prokhorova, Florian Zobel. ${ }^{8}$ These authors jointly supervised this work: Marcin J. Suskiewicz, Ivan Ahel. ${ }^{凶}$ email: marcin.suskiewicz@path.ox.ac.uk; ivan.ahel@path.ox.ac.uk
} 
oly(ADP-ribose) polymerase 1 (PARP1) binding to DNA damage stimulates ADP-ribosylation (ADPr) at numerous sites, both within PARP1 itself and in other proteins ${ }^{1,2}$. PARP1 is assisted by two less abundant paralogs that also catalyse ADPr in response to DNA damage, PARP2 and PARP3 ${ }^{3}$. The ADPr modification events, including poly(ADP-ribose) (PAR) chains of varying length, facilitate DNA repair by promoting chromatin remodelling and recruitment of DNA repair factors ${ }^{4-6}$. In addition to impairing DNA repair, PARP1/PARP2 inhibitors - which are in clinical use against a growing list of cancers-trap the abundant PARP1 protein on DNA breaks, which has genotoxic cytotoxic consequences, especially in BRCA1- or BRCA2deficient cancers $^{7,8}$. PARP1 trapping refers to prolonged residence of bulk PARP1 on damaged chromatin, which might result from physical stalling of PARP1 at DNA breaks but could also consist in continuous recruitment and exchange of PARP1 molecules at these sites ${ }^{9,10}$. Persistence of inhibited PARP1 is the reason why PARP1 inhibition is more detrimental than loss of PARP1 ${ }^{8,11,12}$. Although some inhibitors have additional allosteric properties $^{8,13}$, the available data suggest that PARP1 trapping is caused primarily by the catalytic inhibition of PARP-mediated ADPr, which is needed to terminate PARP1's association with, or persistent recruitment to, chromatin ${ }^{9,14,15}$. However, the ADPr sites that contribute to this process remain unknown. While in vitro studies point to the role of PARP1 auto-modification in preventing the interaction with DNA breaks $8,15-18$ - presumably through steric and electrostatic interference-it is unclear if automodification counteracts trapping in vivo, and, if yes, which exact sites are involved.

With these questions in mind, we turned our attention to serine residues, which we and others have recently revealed to be the main physiological acceptors of ADPr across the human proteome under both basal and DNA-damage conditions, especially if only PARP1- or PARP2-dependent or nuclear ADPr is concerned. The prominence of serine ADPr is reflected both in the number of modification sites detected by mass spectrometry ${ }^{19-24}$ and the amount of ADPr revealed through immunoblotting 24-26. We previously identified histone PARylation factor 1 (HPF1) as a PARP1 and PARP2 regulator essential for efficient serine modification $20,25,27-29$. Structural analysis revealed that HPF1 completes the PARP active site by providing an additional catalytic residue (Glu284 of HPF1) that dictates robust $\mathrm{ADPr}$ initiation reaction at serines ${ }^{29}$. Although a sensitive mass spectrometry approach still detects a number of serine sites in cells lacking $\mathrm{HPF}^{24}$, the loss of HPF1 results in a specific $\sim 200$-fold reduction of DNA damage-induced serine ADPr, rendering non-serine $\mathrm{ADPr}$ relatively more prominent. Indeed, the major modification events in the absence of HPF1 visualised with anti-ADPr immunoblots appear to be localised to glutamate and aspartate residues, as judged by their hydroxylamine sensitivity ${ }^{25}$. In addition to positive dependence on HPF1, serine ADPr is negatively regulated by the serine-specific ADP-ribosylhydrolase 3 (ARH3) ${ }^{30,31}$, the deletion of which results in an $\sim 100$-fold increase in basal modification level of serine sites ${ }^{24}$. Possible other candidates for regulation by the HPF1/ARH3 axis include chemically similar but far rarer ADPr events on tyrosine and threonine residues, although only the connection between the first of them and HPF1 has been established so $\mathrm{far}^{32,33}$. Other ADPr types, including glutamate and aspartate and also cysteine, histidine, or arginine attachments, clearly do not correlate with HPF1 and ARH3 presence/loss in the manner in which serine ADPr does ${ }^{24}$. This indicates that they are not regulated by these factors and, indeed, except for acidic residues, most likely are not dependent on PARP1 or PARP2.

Here, we demonstrate that globally altering serine ADPr levels through manipulation of HPF1 or ARH3 levels affects PARP1 residence on chromatin, PARP1/PARP2 inhibitor sensitivity, and inhibitor-mediated synthetic lethality with BRCA1/BRCA2 deficiencies. We also identify serine residues 499,507 and 519 as the predominant in vivo PARP1 auto-modification sites and demonstrate that preventing their modification through mutation is sufficient to prolong PARP1 retention on DNA damage and sensitise cells to PARP1/PARP2 inhibitors. Altogether, our data connect for the first time PARP trapping and PARP1/PARP2 inhibitor response with specific ADPr sites and implicate HPF1 and ARH3 protein levels as potential biomarkers that predict vulnerability or resistance to therapies based on PARP1/PARP2 inhibition.

\section{Results}

HPF1 loss enhances PARP1/PARP2 inhibitor sensitivity and PARP-BRCA synthetic lethality. To investigate whether, and through what mechanism, HPF1 and serine ADPr impact the PARP inhibitor response, we first tested sensitivity of cells lacking HPF1 to various PARP inhibitors that differ in their specificity and pharmacological properties. To this end, we used several clinically relevant PARP1- and PARP2-specific inhibitors ${ }^{34}$ (Olaparib, Talazoparib, Veliparib, Niraparib and Rucaparib) and, as a control, compared them with the PARP3 inhibitor ME0328 ${ }^{35}$, the PARP5a/PARP5b (TNKS1/TNKS2) inhibitor XAV-93936, and the PARG inhibitor PDD00017273 37 . By performing a long-term colony formation assay, we observed marked sensitisation to all PARP1/PARP2 inhibitors in U2OS cells upon the loss of HPF1 (Fig. 1a and Supplementary Fig. 1a). This is consistent with the established role of HPF1 in selectively regulating these two PARPs ${ }^{27,29}$ and our previous observation of HPF1 knockout (KO) cell sensitivity to Olaparib ${ }^{27}$. The specificity of this effect is confirmed by the fact that HPF1 deletion did not significantly impact sensitivity to PARP3, PARP5a/b, or PARG inhibitors (Supplementary Fig. 1a-c). Importantly, as shown before $^{27}$, simultaneous deletion of $H P F 1$ and $P A R P 1$ largely abolished HPF1 KO sensitivity to Olaparib (Supplementary Fig. 1d), supporting the dependence of the inhibitor-induced survival defect in HPF1 KO cells on PARP1 inhibition and/or trapping.

Loss-of-function mutations in either BRCA1 or BRCA2 lead to defects in double-strand break (DSB) repair by homologous recombination $(\mathrm{HR})^{38,39}$ and also render cells hypersensitive to PARP1/PARP2 inhibition ${ }^{40,41}$. PARP1 plays a key role in singlestrand break (SSB) repair ${ }^{42}$ and has been suggested to be retained primarily at SSBs upon PARP1/PARP2 inhibitor treatment ${ }^{7}$. After being encountered by the transcription machinery or a replication fork, DNA-bound PARP1 is thought to trigger DSB formation ${ }^{7}$. These DSBs can then be resolved by functional HR, a major pathway for DSB repair in S-phase, but can only be repaired by alternative, less efficient and error-prone pathways in HR-defective BRCA1- or BRCA2-deficient cells. Given that above, upon HPF1 loss, we observed a striking cell sensitivity to PARP1/PARP2 inhibition similar to that in HR-defective cells, we next tested whether deleting HPF1 would enhance the effect of PARP1/PARP2 inhibition on cells with BRCA1 or BRCA2 deficiency. To that end, U2OS WT or HPF1 KO cells were transfected with control, BRCA1 or BRCA2 siRNAs and assessed for survival in response to PARP1/PARP2 inhibition. As expected, knockdown of $B R C A 1$ or BRCA2 sensitised WT cells to Olaparib. However, when $B R C A$ downregulation was combined with HPF1 deletion, inhibitor sensitivity was even larger, with complete killing achieved with as little as $1 \mathrm{nM}$ Olaparib for $B R C A 1$ knockdown (Fig. 1b and Supplementary Fig. 2a). The loss of HPF1 thus enhances the synthetic lethality between PARP1/ PARP2 inhibition and BRCA1/BRCA2 deficiency, suggesting that 
a
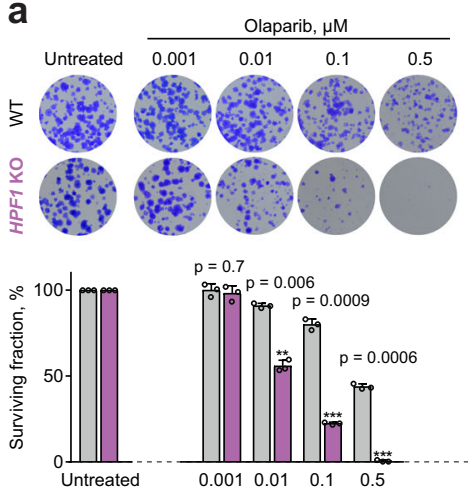

$p=0.7$

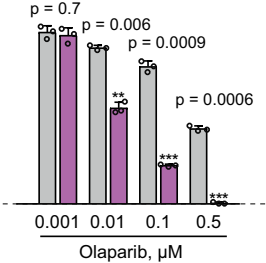

b
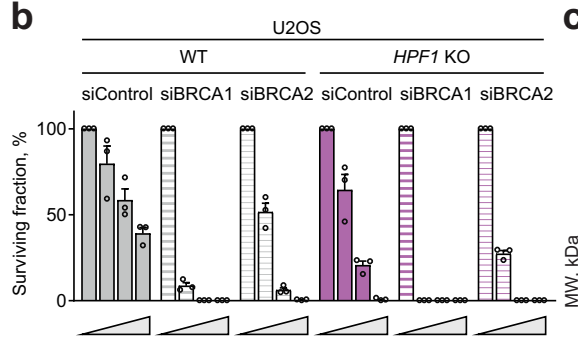

Olaparib: 0, 0.001, 0.01, $0.1 \mu \mathrm{M}$

d

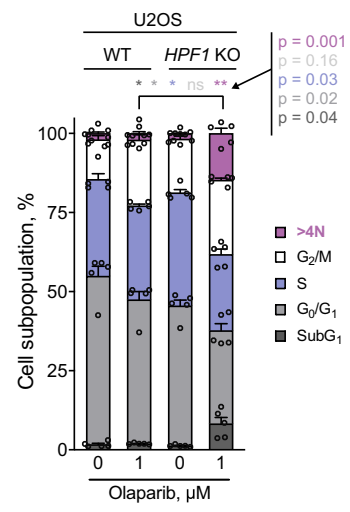

C

e
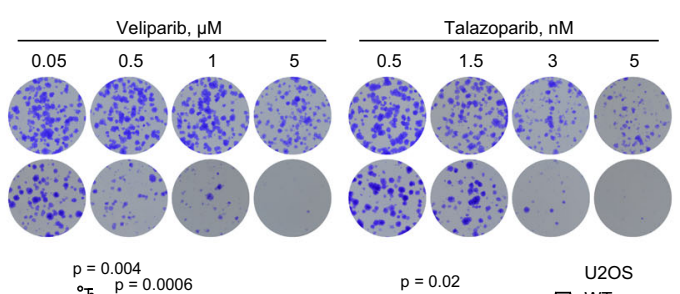

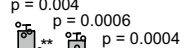
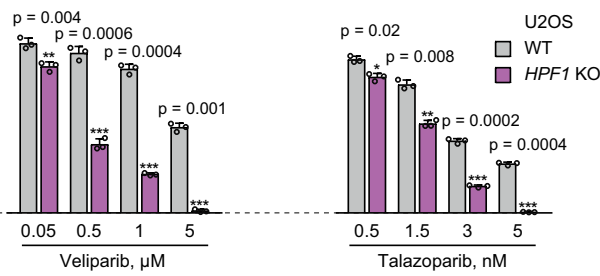

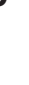
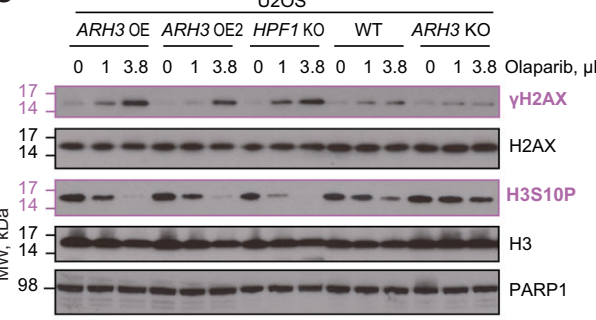

Q็ 17
文 14
98

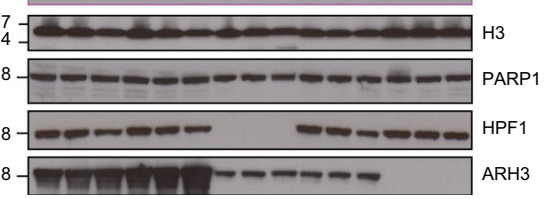$$
38-
$$

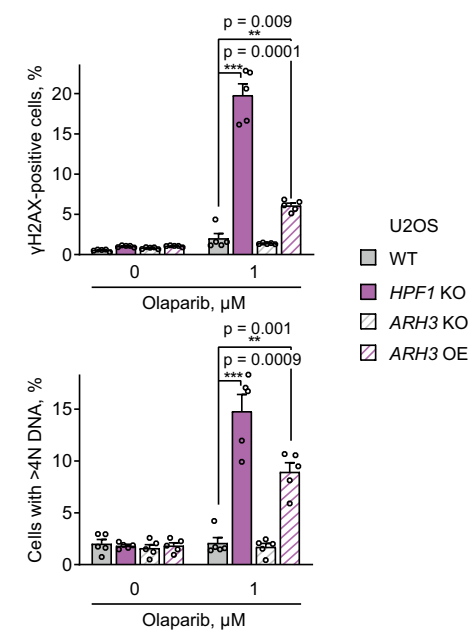

f
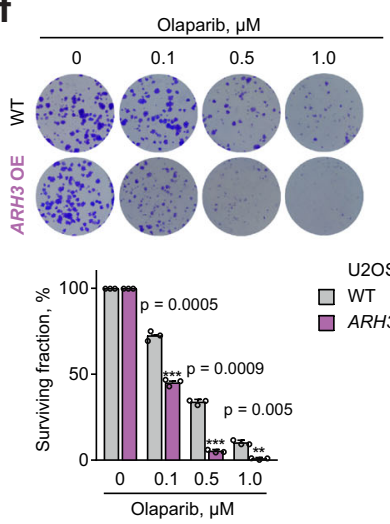

g
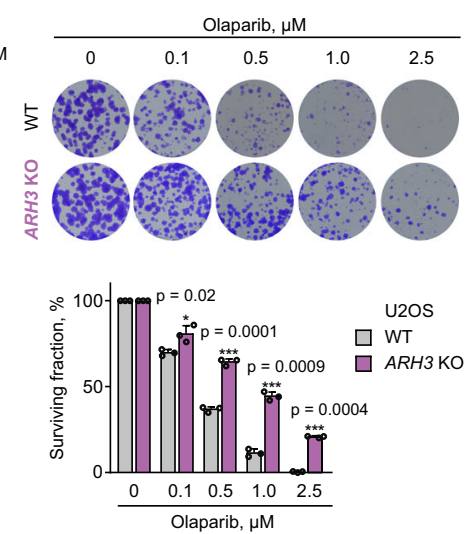

h
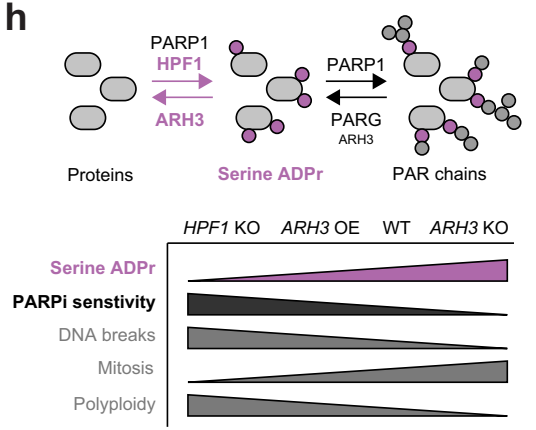

Fig. 1 PARP1/PARP2 inhibitor sensitivity is determined by serine ADPr levels, which are controlled by HPF1 and ARH3 activity. a Reduced survival of HPF1 KO cells after treatment with the indicated PARP1/PARP2 inhibitors. Representative images (top) and quantification of colony formation assay (bottom). b HPF1 loss results in further sensitisation of BRCA1- or BRCA2-deficient cells to Olaparib. See Supplementary Fig. 2a for BRCA1, BRCA2, HPF1 and tubulin control immunoblots. c Effects of 6-day Olaparib treatment on $\gamma \mathrm{H} 2 \mathrm{AX}$ formation and H3S1OP reduction depend on cellular HPF1 and ARH3 protein levels. The experiment was repeated independently 3 times with similar results. See Supplementary Fig. $2 b$ for Pan ADPr immunoblot. A repeat of the experiment with an additional concentration of Olaparib is provided in Supplementary Fig. 2c. d Flow cytometry analysis of cell cycle profiles following 4-day exposure to Olaparib. Asterisks in different colours indicate significant difference in corresponding cell populations between WT and HPF1 KO cells. e HPF1 and ARH3 status determines the effects of 4-day Olaparib treatment on $\gamma \mathrm{H} 2 \mathrm{AX}$ levels (top) and percentage of cells with $>4 \mathrm{~N}$ DNA (bottom) as determined by flow cytometry. $\mathbf{f} A R H 3$ overexpression (OE) renders cells more sensitive to PARP1/PARP2 inhibition. $\mathbf{g}$ Loss of ARH3 confers resistance to Olaparib. $\mathbf{h}$ Schematic representation of ADPr synthesis and removal (top), and summary of the impact of HPF1 and ARH3 status on cell serine ADPr levels and PARP1/PARP2 inhibitor (PARPi) sensitivity (bottom). $\mathbf{a}, \mathbf{f}, \mathbf{g}$ Data are shown as mean \pm SD of three independent experiments. $\mathbf{b}, \mathbf{d}, \mathbf{e}$ Data are shown as mean \pm SEM of three $(\mathbf{b})$ or five $(\mathbf{d}, \mathbf{e})$ independent experiments. ${ }^{\star} p<0.05 ;{ }^{\star \star} p<0.01 ;{ }^{\star \star \star} p<0.001$ (two-tailed Student's $t$-test). Flow cytometry gating strategy for the analyses shown in (d) and (e) is shown in Supplementary Fig. 2 d.

the effect of HPF1 deletion lies upstream of the deficiencies in HR repair and might involve protection from PARP1 trapping.

Next, we went on to further characterise the phenotypes of HPF1-deficient cells treated with a PARP1/PARP2 inhibitor. Since these experiments were performed in a shorter time frame than that for the survival assays above (where toxic effects of inhibition can accumulate over time), we applied higher Olaparib concentrations. We first observed that the loss of HPF1 results in increased DSB formation (as marked by increased levels of $\gamma \mathrm{H} 2 \mathrm{AX}$ ) upon Olaparib treatment (Fig. 1c and Supplementary 
Fig. 2b, c). We also examined the cell cycle distribution and found that the loss of HPF1 in Olaparib-treated cells led to accumulation of endoreplicating polyploid cells with $>4 \mathrm{~N}$ DNA content, and decreased cell proliferation as shown by reduced EdU incorporation and increased cell death $\left(\mathrm{SubG}_{1}\right.$ subpopulation) (Fig. 1d, e). Under the same conditions, we also detected a much higher fraction of $\gamma \mathrm{H} 2 \mathrm{AX}$-positive cells (Fig. 1e). These effects indicate that HPF1 loss potentiates the PARP1/PARP2 inhibitorinduced DNA damage load and causes problems with fundamental cellular processes of DNA replication, chromatin segregation and cell division. Similar changes have been associated with the cytotoxic effects of PARP1/PARP2 inhibition in different cells ${ }^{43-45}$, which suggests that the absence of HPF1 aggravates these effects rather than triggering separate events.

Changing serine ADPr levels through ARH3 manipulation modulates PARP1/PARP2 inhibitor sensitivity. HPF1 deficiency dramatically reduces serine-linked ADPr but could also have additional, independent consequences. Therefore, to confirm that PARP1/PARP2 inhibitor sensitivity observed upon HPF1 deletion is related to the impairment of serine ADPr, we suppressed this modification through alternative means, namely by overexpressing $\mathrm{ARH} 3$, the hydrolase that we previously identified as specific for the serine-ADP-ribose linkage ${ }^{31}$. Strikingly, stable $A R H 3$ overexpression (OE) in U2OS cells, despite having no effect on HPF1 or PARP1 protein levels (Fig. 1c and Supplementary Fig. 2c), resulted in very similar effects to those observed upon HPF1 KO. These include exacerbated sensitivity to PARP1/PARP2 inhibitors (demonstrated for Olaparib) (Fig. 1f), as well as a marked increase in $\gamma \mathrm{H} 2 \mathrm{AX}$ formation (Fig. 1c and Supplementary Fig. 2c) and in the percentage of cells with $>4 \mathrm{~N}$ DNA content and $\gamma \mathrm{H} 2 \mathrm{AX}$-positive cells (Fig. 1e) upon Olaparib treatment. In addition, we observed a significant decrease in H3 serine 10 phosphorylation levels, a marker of cells that undergo mitosis, in both HPF1 $\mathrm{KO}$ and $A R H 3$-overexpressing cells following treatment with Olaparib (Fig. 1c). This suggests that both of these cell lines were unable to enter mitosis and instead continued with DNA replication as manifested by an increased number of polyploid cells.

We hypothesised that if the absence of, or strong reduction in, serine-linked ADPr is a major cause of PARP1/PARP2 inhibitor sensitivity, then, conversely, globally increasing the levels of this post-translational modification might cause PARP1/PARP2 inhibitor resistance. Consistent with this hypothesis, the loss of ARH3 in U2OS cells, which elevates serine-linked ADPr levels $24,25,31$, resulted in a marked resistance to Olaparib when compared to WT cells (Fig. 1g). Moreover, upon Olaparib treatment, these cells showed almost no formation of $\gamma \mathrm{H} 2 \mathrm{AX}$ (Fig. 1c), induction of polyploidy (Fig. 1e), or decrease in the levels of mitotic cells (Fig. 1c). Overall, these results strongly support a model whereby PARP1/PARP2 inhibitor susceptibility is controlled by the levels of serine-linked ADPr and wherein HPF1 and ARH3 play indirect roles by regulating this modification (Fig. 1h).

HPF1-dependent changes in PARP1 auto-modification levels correlate with PARP1/PARP2 inhibitor sensitivity. The changes in modification that we triggered by manipulating HPF1 and ARH3 would globally apply to all or most of the thousands of serine (and possibly other hydroxyl) ADPr sites that can be detected in cells. However, the observed sensitivity profiles are most likely related to a (small) subset of these sites that are mechanistically involved in counteracting PARP1 trapping. The well-established role of PARP1 auto-modification in regulating PARP1-DNA interaction in vitro ${ }^{8,15-18}$ points to serine sites within PARP1 as possible candidates. Moreover, PARP1 automodification accounts for a major fraction of the total proteinlinked $\mathrm{ADPr}^{25}$ (Supplementary Fig. 6) and it is more conceivable that bulk behaviour of a very abundant protein such as PARP1 is regulated by an abundant modification. Before directly testing the importance of serine-linked auto-modification for limiting PARP1 trapping and improving PARP1/PARP2 inhibitor tolerance, we first asked if changes in PARP1 auto-modification levels in the presence and absence of HPF1 at various molarities of inhibitors correlate with observed sensitivity profiles. Importantly, PARP1 is significantly auto-modified both in the presence and absence of HPF1. In the latter situation the auto-modification appears to be primarily on glutamate and aspartate residues ${ }^{25}$, although it also includes inefficiently modified serine sites ${ }^{24}$. The existence of such HPF1-independent auto-modification allows the proposal of a unifying mechanism for preventing trapping that could explain why HPF1 deficiency is normally well-tolerated and only becomes toxic when cells are challenged with PARP1/ PARP2 inhibition. Consistent with this idea, we hypothesised that various forms of PARP1 auto-modification are functionally equivalent and there might be a minimal threshold of automodification that is required for cell survival. If that is the case, then HPF1-independent auto-modification should be brought below this level with lower PARP1/PARP2 inhibitor concentrations compared to HPF1-dependent serine ADPr. To test this model, we monitored ADPr levels in 293T cells with increasing molarities of Olaparib (Fig. 2a). In these cells, endogenous ADPr is more easily detected in the absence of exogenous stimuli compared to U2OS cells ${ }^{25}$, which allowed to perform the experiment without stimulating ADPr using exogenous DNA damaging agents. Whereas for WT cells, ADPr was relatively high in the absence of inhibitors and could be detected on PARP1, as well as histones and other substrates, in HPF1 KO cells ADPr under the same conditions was markedly lower and appeared limited only to PARP1 auto-modification. Moreover, upon addition of as little as $0.1 \mu \mathrm{M}$ Olaparib, the ADPr signal in HPF1 $\mathrm{KO}$ cells was no longer detectable. On the other hand, in WT cells ADPr was only fully lost with $10 \mu \mathrm{M}$ Olaparib. Next, we transiently overexpressed FLAG-tagged HPF1 in HPF1 KO cells, which increased HPF1 levels far above its normal low cellular abundance. This procedure caused a robust PARP1 automodification signal that persisted even at high inhibitor concentrations. Similar effects of manipulating HPF1 levels on PARP1 auto-modification were seen when titrating Talazoparib (Supplementary Fig. 3a). In a follow-up experiment, significant auto-modification of PARP1 in the presence of Olaparib was observed in 293T WT cells when overexpressing WT HPF1 but not HPF1 mutated in the critical catalytic residue, Glu284, or a control PAR-binding protein, APLF (Fig. 2b). Overall, these data demonstrate that the auto-modification synthesised by PARP1 in the presence of catalytically competent HPF1 requires higher inhibitor concentrations to be suppressed compared to automodification produced by PARP1 alone, consistent with the increased PARP1/PARP2 inhibitor sensitivity of HPF1-deficient cells.

To further explore this potential mechanism, we probed the effect of exogenous DNA damage and of deleting the autoinhibitory helical subdomain (HD) of PARP1 on the ADPr signal detectable under Olaparib treatment (Fig. 2c). Both damage induction and $\mathrm{HD}$ deletion $(\triangle \mathrm{HD})$ have a dual consequence: they activate PARP ${ }^{46}$ while also enhancing its affinity for HPF1 (which is relatively low for PARP1's unactivated form ${ }^{29}$ ). We monitored ADPr in 293T WT cells in the presence of $1 \mu \mathrm{M}$ Olaparib, a concentration that in the above Olaparib-titration experiment led to a very low, but still detectable PARP1 automodification signal without any overexpressed proteins. We 

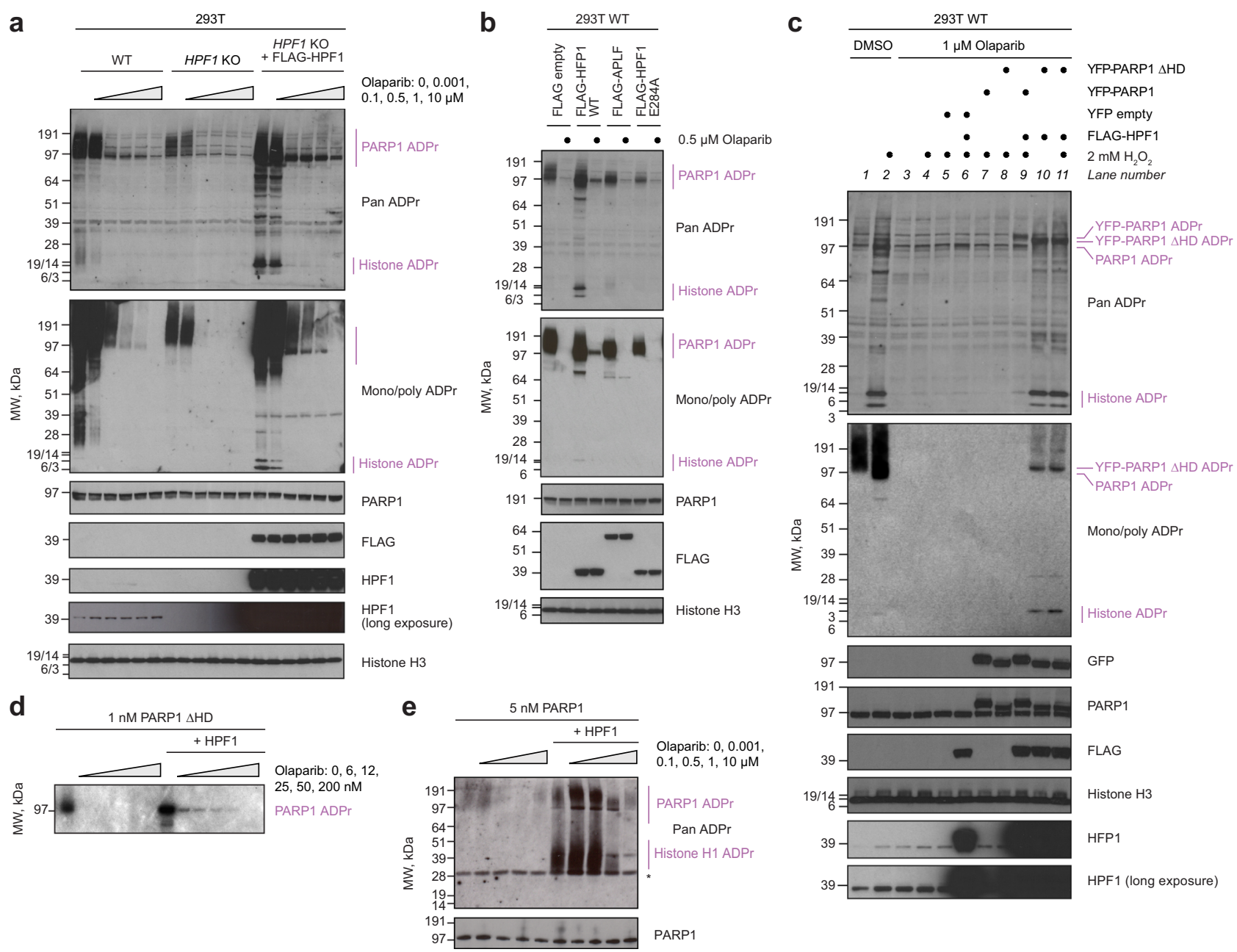

Fig. 2 Serine ADPr persists at high doses of PARP1/PARP2 inhibitor. a Cellular ADPr levels detected throughout increasing molarities of Olaparib depend on HPF1 status. b Overexpression of FLAG-HPF1 WT but not catalytic mutant E284A leads to ADPr that persists despite Olaparib treatment. $\mathbf{c}$ High ADPr levels achieved by YFP-PARP1 $\triangle \mathrm{HD}$ overexpression despite Olaparib treatment depend on simultaneous FLAG-HPF1 overexpression. ADPr levels are also increased above basal levels for this Olaparib concentration by DNA damage $\left(\mathrm{H}_{2} \mathrm{O}_{2}\right)$, FLAG-HPF1 overexpression, or simultaneous YFP-PARP1 and FLAG-HPF1 overexpression. A similar experiment in PARP1 KO cells is shown in Supplementary Fig. 3b. $\mathbf{d}$ In vitro radioactive ADPr assay shows ADPr can be detected at higher Olaparib molarities when HPF1 is present in the reaction. $\mathbf{e}$ In vitro ADPr assay with higher NAD+ concentration than in (d) and histone $\mathrm{H} 1$ as a substrate. Both PARP1 and histone $\mathrm{H} 1 \mathrm{ADPr}$ can be detected at relatively high Olaparib molarities when HPF1 is present. Asterisk indicates non-specific recognition of unmodified histone $\mathrm{H} 1$ by the Pan ADPr reagent. In the Pan ADPr blots in (a) and (c), the weak bands recurrent across all conditions represent a background against which specific signal should be interpreted. a-e The experiments were performed independently at least 2 times with similar results.

observed that this signal, detected especially with anti-Pan ADPr reagent, could be increased slightly by hydrogen peroxide $\left(\mathrm{H}_{2} \mathrm{O}_{2}\right)$-induced DNA damage (lane 4), and further by simultaneous transient overexpression of FLAG-tagged HPF1 (lane 6), consistent with the low endogenous levels of HPF1 being the limiting factor ${ }^{27}$. Conversely, overexpressing YFP-PARP1, either WT or $\triangle \mathrm{HD}$, without simultaneous HPF1 co-expression did not result in any additional signal compared to DNA damage alone (lanes 7 and 8), despite the fact that PARP1 $\triangle H D$ is constitutively hyperactive in vitro ${ }^{46}$. However, when FLAG-HPF1 was simultaneously co-expressed with WT or $\triangle \mathrm{HD}$ YFP-PARP1, the resultant HPF1-PARP1 complexes produced significant automodification despite high Olaparib concentration (lanes 9-11). Artificially improving PARP1 binding to HPF1 through HD deletion leads to more complete saturation of PARP1 with $\mathrm{HPF}^{29}$, which-combined with a possible higher activity of this mutant-could explain a particularly striking effect with PARP1 $\Delta$ HD. Similar results were obtained in 293T PARP1 KO cells
(Supplementary Fig. 3b), excluding the possibility that the presence of endogenous PAPR1 could affect the results above. Overall, these data support a model in which PARP1 in combination with HPF1 is able to produce PARP1 automodification levels that are still significant at much higher doses of PARP1 inhibitor compared to PARP1 alone.

HPF1 counteracts PARP1/PARP2 inhibition by stimulating PARP1 auto-modification. The difference in abundance of HPF1-dependent and -independent PARP1 auto-modification in untreated cells and under inhibitor treatment could result from intrinsic properties of PARP1 with and without HPF1, or could be related to how these different ADPr types are processed by cellular hydrolases. For example, a modification that is hydrolysed to a smaller extent would be more abundant and thus require more inhibitor to be suppressed even if it was synthesised with equal efficiency. To approach this question, we performed in vitro ADPr assays looking at the initial stage of the PARP1 
auto-modification reaction in the presence or absence of HPF1 and with increasing molarities of Olaparib but without any additional factors, such as hydrolases, that would be present in the cells. We tested separately the PARP1 $\triangle$ HD-HPF1 complex (using radioactivity-based detection, Fig. 2d) and the full-length PARP1-HPF1 complex in the presence of histone $\mathrm{H} 1$ as an additional substrate (using anti-Pan ADPr reagent-based detection, Fig. 2e). Consistent with in vivo results, both PARP1 automodification and trans-modification of a histone substrate were stimulated by HPF1 in vitro. Moreover, ADPr produced in the presence of HPF1 was detectable at higher Olaparib concentrations. These results are consistent with in vivo data presented above and suggest that the observed effect is at least partially independent of hydrolysis and is related to how PARP1 synthesises ADPr when assisted by HPF1 or acting alone.

The fact that HPF1-dependent PARP1 auto-modification, compared to its HPF1-independent form, requires higher levels of inhibitor to be suppressed could be explained in two nonmutually exclusive ways. In the first scenario, it might be due to higher abundance of this modification. Indeed, we showed above that this is the case in vivo and can be attributed at least in part to higher efficiency of synthesis. Because PARP1 is more active when assisted by HPF1, even when inhibited to the same relative extent by a given amount of inhibitor, it produces more modification. Additionally, the observed effect could be amplified by changes in binding properties of PARP1 in the presence of HPF1: decreased affinity for inhibitors and/or increased affinity for the substrate $\mathrm{NAD}^{+}$, both of which would result in inhibitors being relatively disfavoured in their competition with $\mathrm{NAD}^{+}$. In this second scenario, the same amount of inhibitor would decrease the starting activity of PARP1 to a lower relative extent in the presence of HPF1 compared to its absence. To explore this additional possibility, we developed a fluorescence anisotropybased binding assay. First, we characterised the binding of the fluorescent Olaparib derivative, PARPi-FL ${ }^{47}$, to PARP1 in the presence and absence of HPF1. As the binding proved too strong for directly measuring the dissociation constant $\left(K_{\mathrm{D}}\right)$ in an equilibrium binding experiment, we separately analysed the association (Supplementary Fig. 4a-c) and dissociation (Supplementary Fig. $4 \mathrm{~d}$ ) kinetics and calculated $K_{\mathrm{D}}$ using rate constants (Supplementary Table 1). This approach was the same as that recently published ${ }^{48}$ and led to similar results, indicating that PARPi-FL binds similarly or slightly tighter to PARP1-HPF1 compared to PARP1 alone. Subsequently, we performed equilibrium competition assays, in which PARPi-FL was displaced by increasing molarities of Olaparib or Talazoparib (Supplementary Fig. 4e and Supplementary Table 2). Our results indicate that these inhibitors, too, bind similarly or slightly tighter to the PARP1-HPF1 complex compared to free PARP1, in line with values obtained by the Luger group using a different method ${ }^{48}$. Combined with the recent report of similar Michaelis constant $\left(K_{\mathrm{M}}\right)$ values of PARP1 for $\mathrm{NAD}^{+}$in the presence and absence HPF $1^{49}$, these results speak against altered PARP1 binding properties as an explanation for the apparent inhibitor resistance seen in our experiments in the presence of HPF1. This effect appears to be primarily attributable to the high efficiency with which serine ADPr is synthesised by the PARP1-HPF1 complex, allowing significant levels of modification to break through high inhibitor concentrations.

HPF1-dependent serine-linked PARP1 auto-modification counteracts PARP1 trapping. We next turned to studying the importance of serine ADPr and specifically serine-linked PARP1 auto-modification for preventing PARP1 trapping. To directly test if serine ADPr, first considered globally and not on specific sites, contributes to the detachment of PARP1 from DNA damage sites, we analysed the transient accumulation of PARP1 at damaged chromatin in the presence or absence of HPF1. By tracking endogenous PARP1 using GFP-tagged PARP1 chromobody in live-cell microscopy, we demonstrated markedly slower dissociation of PARP1 from laser-irradiated chromatin in HPF1 KO compared to WT U2OS cells (Fig. 3a-c and Supplementary Fig. 5a). This observation is consistent with a previous result obtained using the ectopically expressed YFP-tagged PARP1 ${ }^{27}$. An HPF1-dependent difference in PARP1 dissociation kinetics was also clearly observed in the presence of 30 and $100 \mathrm{nM}$ Olaparib, which falls in the intermediate concentration range for which in the above experiments HPF1 boosted cell survival and prevented complete suppression of PARP1 automodification. Performing this analysis with 10 or $30 \mu \mathrm{M}$ Olaparib, very high concentrations that are expected to fully inhibit PARP1 independently of the HPF1 status, led to the same effect in both HPF1 KO and WT cells, with PARP1 remaining associated with damage. Similar results were observed when testing intermediate and high Talazoparib concentrations (Supplementary Fig. 5b). These data show that, in the absence of HPF1, PARP1 is more slowly mobilised both without PARP1/PARP2 inhibitors and with clinically relevant intermediate doses of these drugs. Considering the toxic consequences of PARP1 trapping, these differences between WT and HPF1 KO cells could explain the dramatic sensitivity of the latter to PARP1 inhibitors.

Subsequently, we went on to test the relevance of various PARP1 auto-modification sites for preventing PARP1 trapping, focusing mainly on those in the auto-modification domain, which encompasses the central BRCT domain and a neighbouring unstructured region (Fig. 3d). Before performing live-cell imagining, we characterised which sites account for the largest fraction of PARP1 auto-modification detected with immunoblotting. While there are multiple sites within this segment that have been detected through mass spectrometry $20,23,50,51$ or can be predicted, the importance of none of them has been analysed through mutational studies. We focused on three serine $(499,507$ and 519) and six glutamate residues (471, 484, 488, 491, 513 and 514), exploring their relevance through alanine substitution (Fig. 3e). We first transiently expressed WT, S499/507/519A (3S/A), and E471/484/488/491/513/514A (6E/A) YFP-tagged PARP1 variants in U2OS PARP1 KO cells and monitored ADPr at basal conditions, following exposure to a DNA-damaging agent methyl methanesulfonate (MMS), or upon FLAG-HPF1 overexpression. In all these conditions, the auto-modification signal was relatively similar in cells complemented with WT and 6E/A PARP1 but not in those expressing the 3S/A mutant, which showed a marked decrease in auto-modification. This indicates that the overwhelming amount of PARP1 auto-modification at both basal and DNA-damage conditions locates to these three serine sites and that it is mostly modification of these three sites that is induced in the presence of HPF1. This result is consistent with our previous observation that a major fraction of protein ADPr, including PARP1 auto-modification, is HPF1dependent ${ }^{25}$. All three PARP1 variants are active as demonstrated by their ability to modify histones, with cells overexpressing PARP1 3S/A even showing higher histone ADPr levels, possibly because more $\mathrm{NAD}^{+}$is available for this reaction upon loss of the major PARP1 sites. A further complementation experiment with combinatorial double PARP1 serine mutations, S499/507A, S499/ 519A and S507/519A, demonstrated that the first two sites are the most prominent, with their loss producing a similar automodification defect to that seen for the 3S/A mutant (Supplementary Fig. 7a). We next asked if abolishing the key serine automodification sites is sufficient to enhance PARP1 trapping in HPF1-proficient cells. We conducted this experiment in laser- 
a

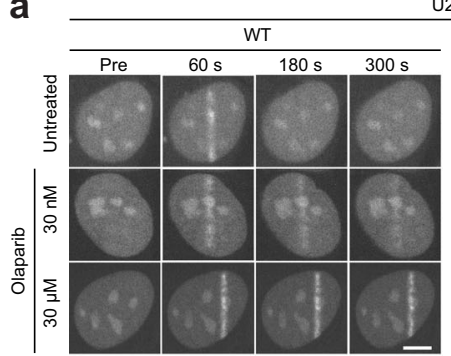

b

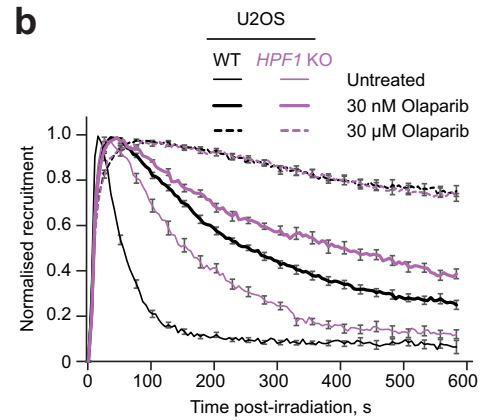

$\mathbf{f}$

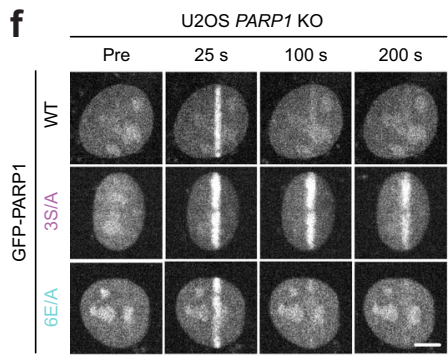

U2OS

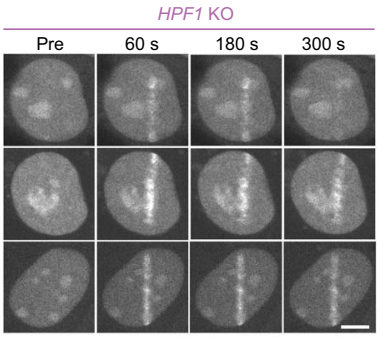

C

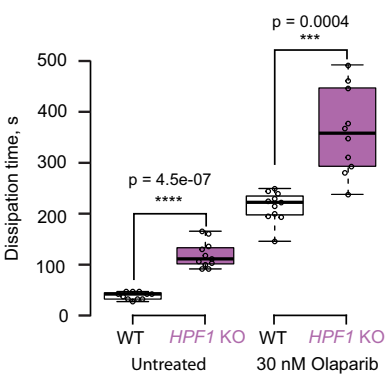

g
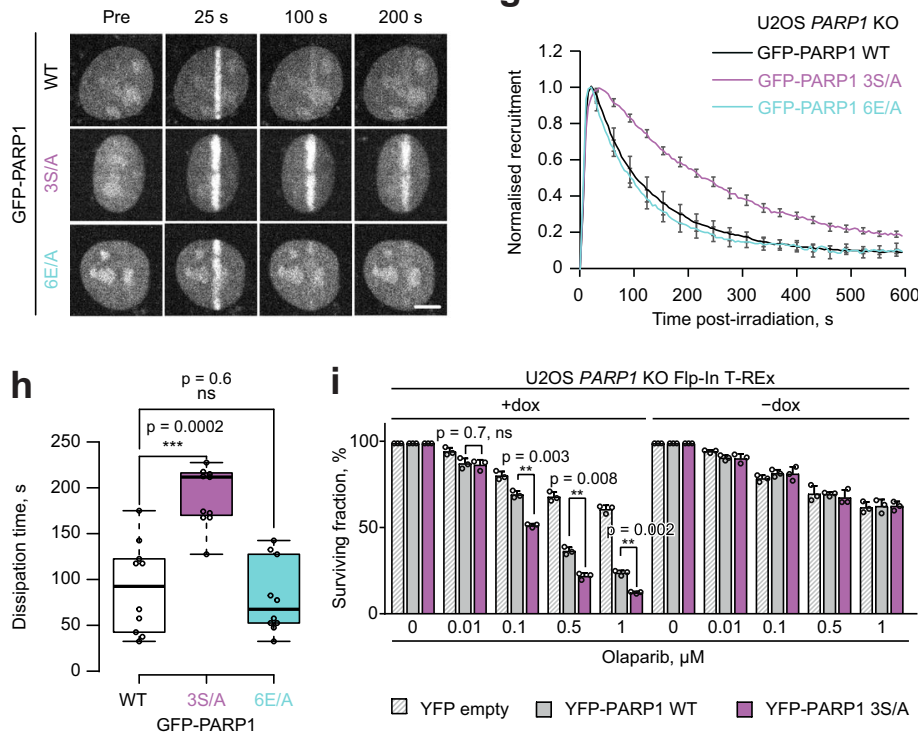

U2OS PARP1 KO FIp-In T-REX

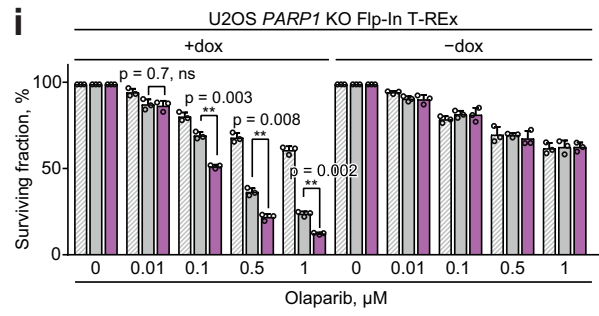

V YFP empty $\square$ YFP-PARP1 WT d

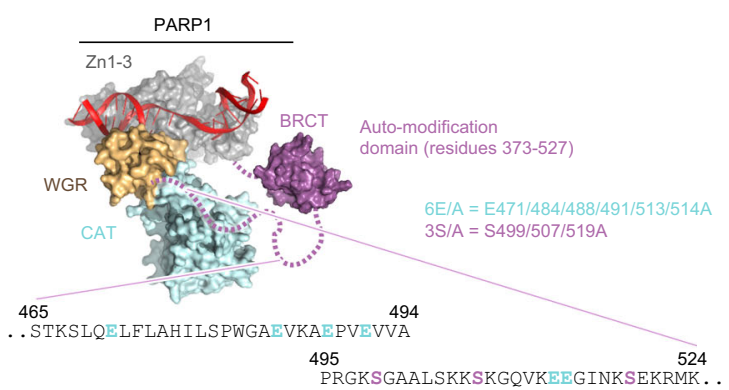

e
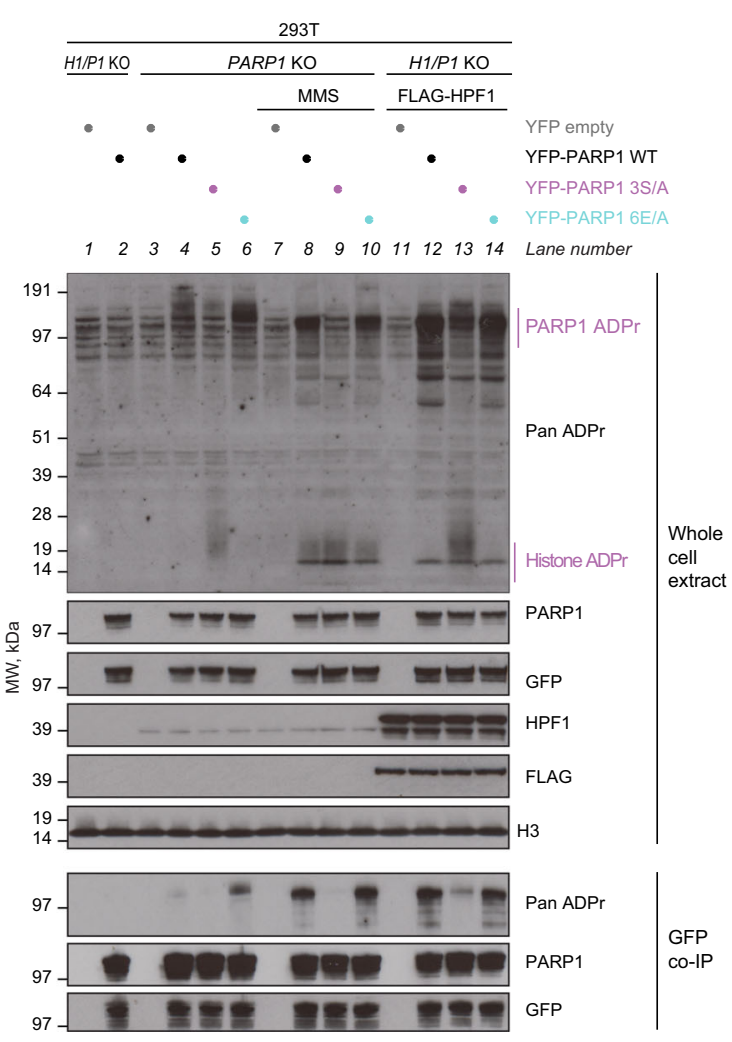

Fig. 3 PARP1 function and release from DNA damage depend on serine ADPr. a Representative confocal images of U2OS WT or HPF1 KO cells transiently expressing GFP-tagged PARP1 chromobody to follow endogenous PARP1 recruitment to sites of laser microirradiation over time in the presence of Olaparib. b Quantification of GFP-PARP1 chromobody accumulation kinetics at damaged sites in WT and HPF1 KO cells. A repeat of the experiment with additional concentrations of Olaparib is provided in Supplementary Fig. 5a. c Dissipation time of GFP-PARP1 chromobody, corresponding to the time required to dissipate $50 \%$ of the maximum PARP1 signal. $\mathbf{d}$ Model of PARP1 (surface representation coloured according to domain composition) bound to a single-strand DNA break (red ribbon) created by alignment of structures from PDB accessions 4DQY, 2N8A and 2LEO. The auto-modification fragment missing from the structures is shown schematically and its sequence containing ADPr sites mutated in point (e) is provided. e Mutation in key serine (S499/507/519A, 3S/A) but not glutamate PARP1 auto-modification sites (E471/484/488/491/513/514A, 6E/A) reduces the levels of endogenous and DNA damage (MMS)- or FLAG-HPF1 overexpression-induced PARP1 ADPr but increases the levels of histone ADPr. P1/H1 KO corresponds to PARP1/HPF1 $\mathrm{KO}$ cells. Representative immunoblots of whole-cell extracts and GFP coimmunoprecipitation (GFP co-IP) samples from three independent experiments are shown. $\mathbf{f}$ Representative confocal images of GFP-PARP1 WT, 3S/A or 6E/A recruitment to sites of laser microirradiation over time. $\mathbf{g}$ Quantification of the accumulation kinetics of GFP-PARP1 WT, 3S/A or 6E/A at damaged sites. $\mathbf{h}$ Dissipation time of GFP-PARP1 WT, 3S/A or 6E/A. i Loss of key serine automodification sites in PARP1 3S/A mutant leads to increased cell sensitivity to Olaparib. Data are shown as mean \pm SEM of three independent experiments. See Supplementary Fig. 7b for GFP, PARP1 and H3 control immunoblots. In (a) and (f), the scale bar represents $5 \mu \mathrm{m}$. In (b) and (g), individual curves were normalised to maximum recruitment; data are shown as mean \pm SEM from the analysis of at least 10 nuclei. In (c) and (h), the box limits correspond to the 25th and 75th percentiles and the bold line indicates the median value; the whiskers extend 1.5 times the interquartile range. ${ }^{*} p<0.05 ;{ }^{* \star} p<0.01$; ${ }^{\star \star \star} p<0.001$ (two-tailed Student's $t$-test). Experiments shown in (a, e, f) were repeated independently at least 3 times with similar results. 
irradiated U2OS PARP1 KO cells by tracking GFP-tagged PARP1 $\mathrm{WT}, 3 \mathrm{~S} / \mathrm{A}$, or $6 \mathrm{E} / \mathrm{A}$ using live-cell microscopy in the absence of inhibitors (Fig. 3f). Consistent with the primary importance of the serine sites, the 3S/A mutation, but not the $6 \mathrm{E} / \mathrm{A}$ one, resulted in an impaired mobilisation compared to WT (Fig. 3g, h). As a control, we also mutated a previously identified tyrosine ADPr site within PARP1 (Y634A) and tested its residence at DNA damage, observing no impact on dissociation kinetics (Supplementary Fig. 5c). Finally, to compare the effects of global serine ADPr loss through HPF1 deletion and targeted ablation of the identified serine PARP1 auto-modification sites in the same experiment, we used live-cell microscopy to monitor recruitment of WT or 3S/A GFP-PARP1 to DNA damage in laser-irradiated U2OS PARP1 KO or double PARP1/HPF1 KO cells without inhibitors (Supplementary Fig. 5d). Mobilisation of WT GFPPARP1 from damage in HPF1-deficient cells was more impaired than that of 3S/A GFP-PARP1 in HPF1-proficient cells, suggesting that while the modification of PARP1 serines 499, 507 and 519 makes a major contribution to preventing PARP1 trapping, there are further HPF1-dependent sites-in or outside PARP1 - that also contribute to this process. It is also worth noting that the HPF1-deficient cells show similar trapping of WT and 3S/A PARP1, confirming that the three identified serine sites are mostly HPF1-dependent.

Loss of serine-linked PARP1 auto-modification sensitises cells to PARP1/PARP2 inhibitors. In the final stage of this study, we asked whether the identified serine PARP1 auto-modification sites contribute to cellular PARP1/PARP2 inhibitor tolerance. To that end, we complemented U2OS PARP1 KO Flp-In T-REx cells with stably integrated genes encoding YFP-tagged PARP1 (WT or 3S/A) or YFP-only control under doxycycline-inducible promoters (Fig. 3i and Supplementary Fig. 7b). Consistent with previous observations that sensitivity to Olaparib is PARP1dependent ${ }^{8}$, the cells complemented with YFP alone or with any of the PARP1 variants but in the absence of doxycycline were highly resistant to the inhibitor. Induction of PARP1 expression resembling endogenous expression levels resulted in increased Olaparib sensitivity. Importantly, this effect was markedly stronger when PARP1 3S/A was expressed instead of the WT enzyme. Additionally, we monitored $\gamma \mathrm{H} 2 \mathrm{AX}$ levels as a marker for DSBs in these cells. Similarly to what was seen above for cells with globally decreased ADPr, expression of the automodification-deficient PARP1 led to higher levels of DNA damage induced by Olaparib treatment (Supplementary Fig. 7c). These findings further support the protective roles of the identified serine sites upon PARP1/PARP2 inhibitor treatment.

Together, our results demonstrate the importance of serinelinked ADPr for limiting PARP1 trapping and PARP1/PARP2 inhibitor-induced toxicity. A substantial part of that effect can be attributed to three prominent auto-modification sites, serines 499, 507 and 519, which, when mutated, increase PARP1's residence on chromatin and sensitise cells to its inhibitors. To our knowledge, this is the first demonstration of the impact of specific ADPr sites on these processes.

\section{Discussion}

Following the recent identification of protein serine residues as the main physiological acceptor of $\mathrm{ADPr}^{22,25}$ and of HPF1 as a specificity factor that is required to efficiently catalyse this process $^{20,27}$, we now demonstrate the crucial importance of HPF1-dependent serine-linked ADPr-specifically on PARP1 itself-for the PARP1/PARP2 inhibitor response. Our data are consistent with a model whereby cell viability requires a minimal threshold of PARP1 auto-modification, which is more robustly maintained when efficient ADPr attachment to serine residues is enabled by appropriate HPF1 and ARH3 levels. Although PARP1 and PARP2 can still catalyse auto-modification in the absence of HPF1, the chains are then inefficiently initiated (presumably primarily on glutamate and aspartate residues), leading to substantially lower ADPr levels in the absence of inhibitors and to easier suppression in their presence. Of note, in vitro studies with free PARP1 suggest that the initial ADP-ribose attachment in the absence of HPF1 is markedly slower than chain elongation and constitutes the rate-limiting step of the process ${ }^{52}$. HPF1, although presumably acting only at the initiation (i.e. mono(ADP-ribosyl) ation or MAR) level, could therefore also increase the PAR synthesis rate by allowing fast attachments to serine residues that can then, in some cases, be robustly extended by PARP1 or PARP2 alone. Here, we did not investigate the relative importance of mono-, oligo- and poly-ADPr for the PARP1/PARP2 inhibitor response, but it is likely that HPF1 will affect, directly or indirectly, all these processes.

Due to its abundance and high affinity for various types of DNA breaks ${ }^{1}$ and obstructions that arise during DNA replication, such as stalled or collapsed replication forks ${ }^{53}$, PARP1 is at a constant risk of getting trapped on chromatin. This points to a key role for a negative feed-back loop whereby PARP1 activation by DNA breaks leads to ADPr, which in turn triggers timely mobilisation of PARP1 from these breaks. Such a cycle has been proposed before based on in vitro studies, which pointed specifically to PARP1 auto-modification as a mechanism that prevented prolonged association of PARP1 with DNA breaks, presumably through electrostatic interference ${ }^{16}$. According to this model, PARP1/PARP2 inhibitors would cause PARP1 trapping and the accompanying toxic consequences primarily by interfering with PARP1's capacity to self-detach through ADPr. However, it has been unclear to what extent the trapping and detrapping observed in cells-processes the nature of which is still poorly understood ${ }^{9}$ - are related to the changes in the direct PARP1-DNA interaction measured in vitro. Here, we directly demonstrate for the first time the importance of automodification at specific PARP1 sites for counteracting PARP1 trapping and inhibitor-induced toxicity in vivo. While this seems to support the relevance of the negative feed-back loop model, it is likely that PARP1 auto-modification in cells exerts its protective role not (only) through direct electrostatic or steric effects on DNA binding, but (also) by recruitment of factors that promote PARP1 mobilisation through chromatin remodelling ${ }^{10}$, repair of DNA lesions ${ }^{9}$, and possibly other mechanisms.

Although our data support specifically the importance of ADPr on serines 499, 507 and 519 of PARP1, we suspect that the different serine-linked and other (presumably mostly glutamate/ aspartate-linked) auto-modification events are partially redundant in their roles. This redundancy-with most known serine and glutamate/aspartate sites localising to the same automodification segment of PARP1-has likely emerged in evolution to ensure that PARP1 dissociation is a robust process that is not easily compromised by point mutations or variations in HPF1 levels. However, when the system is challenged with PARP1/ PARP2 inhibitors, the primary importance of the identified three major serine sites comes into light. The fact that glutamate/ aspartate ADPr evolved as a 'backup' rather than the main form of PARP1 auto-modification might be related to its less efficient modification and lower chemical stability.

Following recent additional approvals, PARP1/PARP2 inhibitors are currently used against breast, ovarian, pancreatic, and prostate cancers. The important message from this development is that therapeutic benefit has been increased more by identifying new susceptible cancer targets for existing PARP1/PARP2 inhibitors than by developing novel, improved inhibitors. Indeed, the 

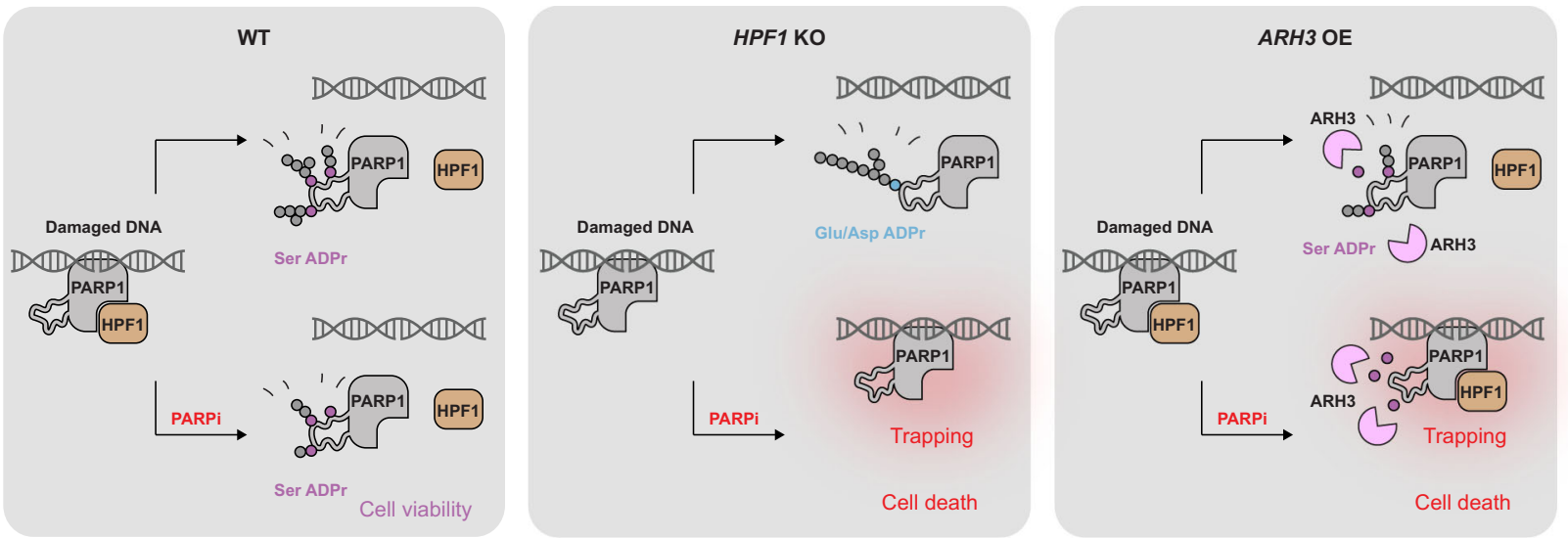

Fig. 4 Proposed model of the effect of HPF1 deficiency and ARH3 overexpression on PARP1/PARP2 inhibitor response. In WT cells, HPF1 allows efficient PARP1 auto-modification at serine residues, especially 499, 507 and 519. This modification is more efficiently produced and therefore partially persists at higher inhibitor concentrations compared to HPF1-independent auto-modification (presumably primarily at glutamate or aspartate residues). In the presence of PARP1/PARP2 inhibitors (PARPi), HPF1-dependent serine auto-modification partially escapes inhibition and promotes cell viability by counteracting PARP1 trapping. This mechanism is compromised in HPF1 KO cells (due to the loss of efficient serine ADPr synthesis) and ARH3 OE (due to higher levels of serine ADPr hydrolysis), leading to PARP1 trapping and cell death upon inhibitor treatment. Endogenous levels of ARH3 in WT and HPF1 KO cells are not indicated.

initial designation of these compounds to BRCA1/BRCA2-mutated cancers already stemmed from the discovery of the synthetic lethal relationship between PARP1/PARP2 inhibition and faulty HR machinery ${ }^{40,41}$. Similarly, our demonstration of synthetic lethality between PARP1/PARP2 inhibition and both HPF1 deficiency and $A R H 3$ OE points to further genetic backgrounds that would sensitise cells to these therapeutics and implicates HPF1 and ARH3 as potential biomarkers for sensitivity/resistance (Fig. 4). In the case of HPF1, one must bear in mind that rather than simply stimulating PARP1, it modulates its specificity, allowing faster initiation at serine residues, while at the same time, as demonstrated previously ${ }^{27,29}$, limiting excessive chain elongation. This suggests that a correct dosage of HPF1 is important for proper balancing of various PARP1- and PARP2catalysed reactions, and therefore subtler changes beyond simple loss-of-function might also be of therapeutic relevance in both cancer and other diseases. Of note, in recent screens, HPF1 was detected as one of the top sensitisers, upon deletion, to PARP1/ PARP2 inhibitors ${ }^{54,55}$, confirming our previous ${ }^{27}$ and new findings. The dramatic susceptibility observed by us for cells with combined HPF1 and BRCA1/BRCA2 deficiencies indicates that PARP1/PARP2 inhibitor therapy might be particularly successful when changes in HPF1 or ARH3 co-exist with other dysfunctions in DNA repair pathways. Importantly, defects in DNA repair are widespread in cancer, with as many as $50 \%$ of ovarian tumours having impaired $\mathrm{HR}^{56}$.

Recently, cellular ADPr levels have been proposed as a biomarker that positively correlates with PARP1/PARP2 inhibitor sensitivity across certain types of cancer cells ${ }^{57-59}$. It is likely, however, that this correlation-which appears to contradict the protective role of $\mathrm{ADPr}-$ indirectly reflects the dependence of inhibitor sensitivity on high PARP1 protein levels and accumulated DNA damage, both of which would manifest as high ADPr levels but enhance PARP1 trapping. We show that if the levels of PARP1 and DNA damage are kept constant by using the same genetic background, ADPr levels-modulated through manipulating secondary ADPr regulators rather than PARP1 protein levels or genome quality-correlate negatively with inhibitor sensitivity, consistent with the model proposed above. The protective role of $\mathrm{ADPr}$ levels clearly emphasised by our study explains the recently reported increased PARP1/PARP2 inhibitor resistance upon downregulation of the hydrolase PARG $^{54,60}$.
Although PARG, unlike ARH3, does not remove the initial attachment on serine residues, it counteracts ADPr synthesis by reversing PAR chain elongation ${ }^{31,61}$.

Our study highlights the embeddedness of PARP1 within a broader network that includes HPF1, ARH3, and PARG, each of which influences PARP1 functions including the negative feedback loop that prevents its toxic trapping on chromatin. Understanding the complex dynamics within this system and the underlying mechanisms is crucial for making the most of the existing, and developing novel, PARP1/PARP2 inhibitor-based therapies.

\section{Methods}

Cell lines. Human U2OS osteosarcoma (ATCC HTB-96) and embryonic kidney 293T (ATCC CRL-3216) cells were acquired from ATCC. U2OS HPF1 KO, U2OS PARP1 KO, U2OS HPF1/PARP1 KO, 293T HPF1 KO, 293T HPF1/PARP1 KO ${ }^{27}$ and $\mathrm{U} 2 \mathrm{OS} A R H 3 \mathrm{KO}^{31}$ cells were generated previously. The cells were grown in DMEM (Sigma-Aldrich) supplemented with 10\% FBS (Gibco) and penicillinstreptomycin $(100 \mathrm{U} / \mathrm{mL}, \mathrm{Gibco})$ at $37^{\circ} \mathrm{C}$ with $5 \% \mathrm{CO}_{2}$. U2OS Flp-In T-REx cells were generated by Daniel Durocher's laboratory ${ }^{62}$; $293 \mathrm{~T}$ PAPR1 KO and U2OS Flp-In T-REx PARP1 KO cells were generated using CRISPR Cas9 genome editing as previously described ${ }^{16}$, using guide RNA sequences complementary to exon 2, PARP-1-sgRNA\#1 (CCACCTCAACGTCAGGGTG) and PARP-1-sgRNA\#2 (TGGGTTCTCTGAGCTTCGT), and the pSpCas9n(BB)-2A-Puro (PX462) V2.0 vector from Feng Zhang (Addgene plasmid \#62987). The cells were grown in DMEM supplemented with $10 \%$ FBS, $50 \mu \mathrm{g} / \mathrm{mL}$ Zeocin (R25001, Thermo Fisher) and $4 \mu \mathrm{g} / \mathrm{mL}$ Blasticidin (ant-bl-1, Invivogen) at $37^{\circ} \mathrm{C}$ with $5 \% \mathrm{CO}_{2}$.

To generate stable cell lines with ARH3 OE, U2OS cells were plated in $6 \mathrm{~cm}$ dishes and transiently transfected with pDEST12.2 ARH3 WT using TransIT-LT1 Transfection Reagent (Mirus Bio) according to the manufacturer's protocol. After $24 \mathrm{~h}$, the cells were transferred into $15 \mathrm{~cm}$ dishes and allowed to grow for $48 \mathrm{~h}$. Then, the media was replaced with complete DMEM supplemented with $1 \mathrm{mg} / \mathrm{mL}$ G-148 solution (Sigma-Aldrich) for 10 days to select for resistant cells integrated with the pDEST12.2 ARH3 constructs. Individual colonies were picked using cloning discs (Sigma-Aldrich), propagated and screened to check for successful integration via PCR. Immunoblotting was performed on positive colonies to check ARH3 protein levels.

To generate PARP1-expressing inducible cell lines, U2OS Flp-In T-REx PARPI KO cells were plated in 6-well plates and cotransfected with YFP empty vector or plasmids encoding YFP-PARP1 WT or 3S/A and the Flp recombinase plasmid pOG44 (in 1:9 ratio) using TransIT-LT1 Transfection Reagent (Mirus Bio) according to the manufacturer's protocol. At $24 \mathrm{~h}$ following transfection, cells were transferred into $15 \mathrm{~cm}$ dishes in DMEM with $10 \%$ FBS; $24 \mathrm{~h}$ later the media was supplemented with $4 \mu \mathrm{g} / \mathrm{mL}$ Blasticidin (Invivogen) and $200 \mu \mathrm{g} / \mathrm{mL}$ Hygromycin B Gold (Invivogen). The media in the dishes was subsequently changed every 2-3 days for two weeks to select for resistant colonies, which were picked using cloning discs (Sigma-Aldrich), propagated and screened following $24 \mathrm{~h}$ incubation with $1 \mu \mathrm{g} / \mathrm{mL}$ doxycycline (Sigma-Aldrich) by fluorescence microscopy and 
immunoblotting. To resemble endogenous PARP1 protein expression levels in the selected clones, YFP empty vector and YFP-PARP1 expression was induced with $0.1 \mu \mathrm{g} / \mathrm{mL}$ doxycycline; YFP-PARP1 3S/A expression was induced with $0.5 \mu \mathrm{g} / \mathrm{mL}$ doxycycline. The media was replenished every 3 days to maintain expression levels in long-term assays.

Chemical compounds. PARP inhibitors Olaparib, Talazoparib and Veliparib were purchased from Cayman Chemical or Enzo Life Sciences; Niraparib, Rucaparib and ME0328 from Stratech Scientific; XAV-939 and PDD00017273 from SigmaAldrich, and were dissolved in dimethyl sulfoxide (DMSO) (Sigma-Aldrich). Methyl methanesulfonate (MMS) and hydrogen peroxide $\left(\mathrm{H}_{2} \mathrm{O}_{2}\right)$ were obtained from Sigma-Aldrich. Concentrations and durations of treatment are indicated in the sections below and in the respective figures.

Colony formation assay. Cells were plated at low densities (700 cells/well for U2OS cells and 900 cells/well for U2OS Flp-In T-REx cells) in 6-well plates and grown in the indicated conditions for 11 days. Cells were fixed and stained with $0.5 \%$ crystal violet in $25 \%$ methanol for $30 \mathrm{~min}$, washed with water and air-dried. Quantification was performed using ImageJ software. The surviving fraction at each dose was calculated after normalisation to the plating efficiency of untreated samples.

siRNA transfection. siRNA transfection was performed using Lipofectamine RNAiMAX (Invitrogen) according to the manufacturer's instructions. Silencer Select Negative Control No. 2 siRNA and siBRCA1 (s458, CAGCUACCCUUCCAUCAUA) were from Ambion (Invitrogen). siBRCA2 (D-003462-04, GAAGAAUGCAGGUUUAAUA) was purchased from Dharmacon.

Cell cycle analysis. Cells were seeded in 6-well plates, treated and incubated with $10 \mu \mathrm{M}$ EdU for $1 \mathrm{~h}$ at the end of treatment. Cells were harvested by trypsinization and labelled using the Click-iT Plus EdU Alexa Fluor 647 Flow Cytometry Assay Kit (Invitrogen) according to the manufacturer's instructions. For the analysis of DSB levels, cells were then stained protected from light with $\gamma \mathrm{H} 2 \mathrm{AX}$ primary antibody (Cell Signaling, 9718S, 1:200) in 1\% BSA in PBS for $1 \mathrm{~h}$ at room temperature, washed once and incubated for $30 \mathrm{~min}$ with Alexa Fluor 488-conjugated goat anti-rabbit secondary antibody (Molecular Probes/Thermo Fisher, A11034, $1: 500)$ in $1 \%$ BSA in PBS. For DAPI staining, cell pellets were resuspended in $1 \mu \mathrm{g} / \mathrm{mL}$ DAPI solution in PBS and incubated for $10 \mathrm{~min}$. Cells were washed in PBS and analysed immediately after staining on Cytoflex LX (Beckman Coulter), using CytExpert version 2.3 (Beckman Coulter) for data collection. Post-acquisition analysis was performed in FlowJo software version 10 (BD Biosciences).

Plasmids and site-directed mutagenesis. Vectors for bacterial expression of fulllength human HPF1 and PARP1 with N-terminal FLAG or YFP tags were previously described ${ }^{27}$. Mammalian expression vectors encoding FLAG-HPF1 E284A and YFP-PARP1 $\triangle \mathrm{HD}^{29}$, pmEGFP-PARP ${ }^{10}$, and FLAG-APLF ${ }^{63}$ were generated previously. PARP1 point mutations were introduced through site-directed mutagenesis PCR using the QuickChange Lightning kit (Agilent). The primers used to introduce these mutations are listed in Supplementary Table 3.

Immunoblotting. The cells were lysed with Triton-X100 lysis buffer $(50 \mathrm{mM}$ Tris$\mathrm{HCl} \mathrm{pH} \mathrm{8.0,100} \mathrm{mM} \mathrm{NaCl,} 1 \%$ Triton X-100) supplemented with $5 \mathrm{mM} \mathrm{MgCl}_{2}$, protease and phosphatase inhibitors (Roche), Olaparib (Cayman Chemical, $1 \mu \mathrm{M}$ for U2OS and $2 \mu \mathrm{M}$ for $293 \mathrm{~T}$ cells) and $1 \mu \mathrm{M}$ PARG inhibitor PDD00017273 (Sigma-Aldrich, $1 \mu \mathrm{M}$ for $\mathrm{U} 2 \mathrm{OS}$ and $2 \mu \mathrm{M}$ for $293 \mathrm{~T}$ cells) at $4{ }^{\circ} \mathrm{C}$. The lysates were incubated with $0.1 \%$ Benzonase (Sigma-Aldrich) for $30 \mathrm{~min}$ at $4{ }^{\circ} \mathrm{C}$ and rotating at $20 \mathrm{rpm}$, centrifuged at $18,400 \mathrm{~g}$ for $15 \mathrm{~min}$, and the supernatants were collected. Protein concentrations were analysed by Bradford Protein Assay (Bio-Rad). Proteins were boiled in $1 \mathrm{x}$ NuPAGE LDS sample buffer (Invitrogen) with TCEP (Sigma-Aldrich), resolved on NuPAGE Novex 4-12\% Bis-Tris gels (Invitrogen), and transferred onto nitrocellulose membranes (Bio-Rad) using Trans-Blot Turbo Transfer System (Bio-Rad). The membranes were blocked in PBS buffer with $0.1 \%$ Tween 20 and $5 \%$ non-fat dried milk for $1 \mathrm{~h}$ at room temperature and incubated overnight with primary antibodies at $4{ }^{\circ} \mathrm{C}$, followed by $1 \mathrm{~h}$ incubation with peroxidase-conjugated secondary anti-mouse (Agilent, P0447, 1:2000) or antirabbit (Agilent, P0399, 1:2000) antibodies at room temperature. Blots were developed using ECL (Invitrogen) and analysed by exposing to films.

The following primary antibodies were used: mouse anti-BRCA1 (EMD Millipore, OP92, 1:1000), mouse anti-BRCA2 (EMD Millipore, OP95, 1:500), rabbit anti-tubulin (Abcam, ab6046, 1:10,000), rabbit anti-Pan ADPr binding reagent (EMD Millipore, MABE1016, 1:1500), rabbit anti-ARH3 (Atlas Antibodies, HPA027104, 1:1000), rabbit anti- $\gamma \mathrm{H} 2 \mathrm{AX}$ (Abcam, ab2893, 1:2000), rabbit antiH2AX (Cell Signaling, 7631S, 1:1000), rabbit anti-H3 (EMD Millipore, 07-690, 1:50,000), rabbit anti-PARP1 (Abcam, ab32138, 1:5000), rabbit anti-H3S10P (Abcam, ab5176, 1:2000), rabbit anti-Poly/mono ADPr (Cell Signaling, 83732S, 1:1000), rabbit anti-HPF1 (DC Biosciences, custom-made against peptide RELPETDADLKRIC, 1:250 or custom-made published previously ${ }^{27}, 1: 1000$ ), rabbit anti-GFP (Abcam, ab290, 1:5000) and mouse anti-FLAG (Sigma-Aldrich, A8592-1MG, 1:50,000).

In vivo ADP-ribosylation assay. The $293 \mathrm{~T}$ cells were transiently transfected with the indicated constructs using Polyfect Transfection Reagent (Qiagen) according to the Qiagen Quick-Start protocol or were left untransfected. For PARP1/PARP2 inhibitor titration experiments, $24 \mathrm{~h}$ after transfection, cells were treated with an inhibitor (Olaparib or Talazoparib) or equivalent amount of DMSO for $12 \mathrm{~h}$. To induce DNA damage, the cells were washed once with DPBS and treated with $2 \mathrm{mM} \mathrm{H}_{2} \mathrm{O}_{2}$ in DPBS for $10 \mathrm{~min}$, or treated with $0.1 \%$ MMS in complete DMEM for $30 \mathrm{~min}$.

In vitro ADP-ribosylation assay. Recombinant human HPF1 was expressed and purified as described previously and full-length PARP1 and PARP1 $\triangle H D$ as described for full-length PARP ${ }^{29}$. Briefly, these proteins were expressed in Rosetta Escherichia coli cells with an $\mathrm{N}$-terminal $\mathrm{His}_{6}$ tag and purified by $\mathrm{Ni}^{2+}$ affinity (both HPF1 and PARP1), followed by anion-exchange (HPF1) or heparin (PARP1) chromatography, and finally by size-exclusion chromatography using a Superdex 200 column (both HPF1 and PARP1). The last step was performed in the buffer 25 mM HEPES, $\mathrm{pH} 8,200 \mathrm{mM} \mathrm{NaCl}, 1 \mathrm{mM}$ EDTA and $0.1 \mathrm{mM}$ TCEP. In the assay shown in Fig. 2d, $1 \mathrm{nM}$ PARP1 $\triangle \mathrm{HD}$ was incubated with $250 \mathrm{nM}$ HPF1 (if indicated), $250 \mathrm{nM}$ activating DNA duplex (5'-ATCAGATAGCATCTGTGCGGCCG CTTAGGG- $3^{\prime}$ and $5^{\prime}$-CCCTAAGCGGCCGCACAGATGCTATCTGAT- ${ }^{\prime}$ ), and indicated molarities of Olaparib in $50 \mathrm{mM}$ Tris, $\mathrm{pH} 7.5,50 \mathrm{mM} \mathrm{NaCl}$. The reaction was started by adding ${ }^{32} \mathrm{P} \mathrm{NAD}^{+}$from Perkin Elmer (50\% cold at the time of use) to the final concentration of $62.5 \mathrm{nM}$, conducted for $3 \mathrm{~min}$ at room temperature, and quenched with SDS-containing gel-loading dye. The samples were analysed by SDS-PAGE and autoradiography. In the assay shown in Fig. 2e, $5 \mathrm{nM}$ PARP1 was incubated with $2 \mu \mathrm{M}$ HPF1 (if indicated), $2 \mu \mathrm{M}$ recombinant human histone $\mathrm{H}^{0}$ (New England BioLabs), and shown molarities of Olaparib in $50 \mathrm{mM}$ Tris, pH 7.5, $50 \mathrm{mM} \mathrm{NaCl}$. The reactions were started by adding $1 \mu \mathrm{M} \mathrm{NAD}^{+}$and allowed to proceed for $3 \mathrm{~min}$ at room temperature before terminating with SDS-containing gel-loading dye. The results were analysed by SDS-PAGE and immunoblotting with the Pan ADPr reagent. Some non-specific recognition of unmodified H1 was observed.

Binding analysis with fluorescence polarisation/anisotropy. Fluorescence polarisation/anisotropy experiments were performed in $25 \mathrm{mM}$ HEPES, pH 7.2, $200 \mathrm{mM} \mathrm{NaCl}$ and $0.1 \mathrm{mM}$ TCEP in black OptiPlate F 96-well plates (Perkin Elmer) at room temperature. The total sample volume was 100,180 or $400 \mu \mathrm{l}$ for $k_{\text {on }}$ and $k_{\text {off }}$ measurements and competition experiments, respectively. We used two different plate readers, SpectraMax M5 from Molecular Devices (settings: excitation/emission wavelengths of $485 / 530 \mathrm{~nm} ; 20$ readings per data point; and high PMT sensitivity) for $k_{\text {off }}$ measurements and PHERAstar FS from BMG LabTech (settings: excitation/emission A/emission B wavelengths of 485/520/520 nm; 200 flashes/well; $0.5 \mathrm{~s}$ settling time; gain for A/B of 1571/1764; focal height adjusted) for $k_{\text {on }}$ measurements and competition experiments. Data collection was performed using SoftMax Pro version 5.01 and PHERAstar software version 4.00 R3, respectively. For $k_{\text {on }}$ measurements, samples containing $1 \mathrm{nM}$ PARPi-FL (Tocris Bioscience), $3 \mu \mathrm{M}$ HPF1 (if indicated) and $3 \mu \mathrm{M}$ DNA duplex (the same as in the ADP-ribosylation assay) were mixed and their fluorescence polarisation measured. We began measuring time from this moment. Then, $1 \mu \mathrm{l}$ of appropriate PARP1 dilution was rapidly mixed in and a time-course of $600 \mathrm{~s}$ with measurements every $10 \mathrm{~s}$ was started. In this set-up, HPF1 was not pre-incubated with PARP1, but we found that including HPF1, which, due to its high concentration, affects polarisation, in the sample prior to adding PARP1 was necessary for obtaining an accurate starting polarisation value. Since HPF1 is 3000x more concentrated than PARPi-FL, PARP1 is very likely saturated much faster with HPF1 than with the inhibitor. For $k_{\text {off }}$ measurements, we pre-incubated $5 \mathrm{nM}$ PARPi-FL with $25 \mathrm{nM}$ PARP1, $3 \mu \mathrm{M}$ HPF1 (if indicated), and $3 \mu \mathrm{M}$ DNA duplex for $10 \mathrm{~min}$ prior to adding $164 \mu \mathrm{M}$ non-fluorescent Olaparib. Polarisation was then monitored every $4 \mathrm{~min}$ over $19 \mathrm{~h}$. For equilibrium competition experiments, we incubated $1 \mathrm{nM}$ PARPi-FL with $6 \mathrm{nM}$ PARP1, $3 \mu \mathrm{M}$ HPF1 (if indicated), $0.5 \mu \mathrm{M}$ duplex DNA, and a range of molarities of the indicated inhibitors for $20 \mathrm{~h}$ prior to measuring polarisation. For all experiments, the measured values were converted to anisotropy. Data from individual experiments were fitted to Eqs. (1), (2) or (3) (as appropriate) by minimising squared errors between actual and predicted values using Excel Solver:

$$
\begin{gathered}
\text {-association: } A=A_{\min }+\left(A_{\max }-A_{\min }\right) \times\left(1-\mathrm{e}^{-k_{\mathrm{on}} \times[\mathrm{PARP}] \times t}\right) \\
- \text { dissociation: } A=A_{\min }+\left(A_{\max }-A_{\min }\right) \times \mathrm{e}^{-k_{\mathrm{off}} \times t} \\
\text { - competition: } A=A_{\min }+\frac{A_{\max }-A_{\min }}{1+10^{\log ([\operatorname{Inhhibitor}])-\log \left(\mathrm{IC}_{50}\right)}},
\end{gathered}
$$

where $A$ is the measured anisotropy, $A_{\min }$ and $A_{\max }$ are the minimal and maximal anisotropy values (set to an empirical value or fitted as judged appropriate), [PARP1] is PARP1 concentration, $t$ is time, $k_{\text {on }}$ and $k_{\text {off }}$ are association and dissociation rate constants, [Inhibitor] is inhibitor (Olaparib or Talazoparib) 
concentration, and $\mathrm{IC}_{50}$ is half-maximal inhibitory concentration. Each experimental repeat was fitted individually and then the mean and $\mathrm{SD}$ values calculated. $K_{\mathrm{D}}$ for the interaction between PARPi-FL and PARP1 was obtained using the formula: $K_{\mathrm{D}}=\frac{k_{\mathrm{on}}^{-}}{k_{\mathrm{off}}}$, and its SD with the formula (4):

$$
\mathrm{SD}=\frac{1}{\sqrt{N}} \frac{k_{\mathrm{on}}^{-}}{k_{\mathrm{off}}} \sqrt{\frac{\mathrm{SD}_{k_{\mathrm{on}}}{ }^{2}}{k_{\mathrm{on}}^{2}}+\frac{\mathrm{SD}_{k_{\mathrm{off}}}{ }^{2}}{k_{\mathrm{off}}{ }^{2}}},
$$

where $k_{\text {on }}^{-}$and $k_{\text {off }}^{-}$indicate the mean values of these rate constants and $\mathrm{SD}_{k_{\mathrm{on}}}$ and $\mathrm{SD}_{k_{\text {off }}}$ represent the SD values of $k_{\text {on }}$ and $k_{\text {off }}$, respectively, while the sample size $N$ $=3$. The inhibitory constant $K_{\mathrm{i}}$ for Olaparib was estimated from $\mathrm{IC}_{50}$ as previously described $^{64}$, using a Microsoft Excel file by Dr. Chao-Yie Yang available at http:// www.umich.edu/ shaomengwanglab/software/calc_ki/index.html. This approach is unreliable for $\mathrm{IC}_{50}$ values close to, equal, or lower than the protein concentration used, precluding the estimation of $K_{\mathrm{i}}$ for Talazoparib.

Live-cell imaging. Cells were seeded into an 8-well imaging chamber (Zell-Kontakt) and transfected with TagGFP2-PARP1 chromobody (Chromotek) and PATagRFP-H2B ${ }^{65}$ or GFP-PARP1 WT or point mutants and PATagRFP-H2B with X-tremeGene HP (Roche) according to the manufactures instructions $48 \mathrm{~h}$ prior to imaging. Cells were sensitised with fresh media containing $0.15 \mu \mathrm{g} / \mathrm{mL}$ Hoechst 33342 for $1 \mathrm{~h}$ at $37^{\circ} \mathrm{C}$. Immediately prior to imaging, the medium was replaced with $\mathrm{CO}_{2}$-independent phenol red-free Leibovitz's L15 medium (Life Technologies) supplemented with $20 \%$ FBS, either with or without PARP1/PARP2 inhibitors. Cells were incubated with PARP inhibitors for a minimum of $30 \mathrm{~min}$ prior to imaging. Live-cell imaging was performed on a Nikon Ti-E inverted microscope equipped with a spinning-disk scan head CSU-X1 from Yokogawa at a rotation speed of $5000 \mathrm{rpm}$, a Plan APO 60x/1.4N.A. oil-immersion objective lens and a sCMOS ORCA Flash 4.0 camera. Laser microirradiation and local photoactivation at $405 \mathrm{~nm}$ was performed along a $16 \mu \mathrm{m}$ line through the nucleus using a single-point scanning head (iLas2 from Roper Scientific) coupled to the epifluorescence backboard of the microscope. To ensure reproducibility, laser power at $405 \mathrm{~nm}$ was measured at the beginning of each experiment and set to $125 \mu \mathrm{W}$ at the sample level. The fluorescence of GFP and PATagRFP were excited with lasers at 490 and $561 \mathrm{~nm}$, respectively. For fluorescence detection, we used bandpass filters adapted to the fluorophore emission spectra. Cells were maintained at $37^{\circ} \mathrm{C}$ with a heating chamber. Protein recruitment was quantified using custom-made MATLAB (MathWorks) routines available in a GitHub repository at https://github.com/ sehuet/Recruitment-break. The characteristic dissociation time corresponds to the time required to dissipate $50 \%$ of the maximum PARP1 signal at the DNA lesion.

Figure preparation. Graphs were prepared in Microsoft Excel 2016 or GraphPad PRISM 7 and further edited in Adobe Illustrator 25.1, which was also used to assemble all figures.

Reporting summary. Further information on research design is available in the Nature Research Reporting Summary linked to this article.

\section{Data availability}

MATLAB routines used to quantify PARP1 recruitment in live-cell imaging experiments are deposited in a GitHub repository at https://github.com/sehuet/Recruitment-break. Any other relevant data are available from the authors. Source data are provided with this paper.

Received: 21 September 2020; Accepted: 17 June 2021; Published online: 01 July 2021

\section{References}

1. D'Amours, D., Desnoyers, S., D'Silva, I. \& Poirier, G. G. Poly(ADP-ribosyl) ation reactions in the regulation of nuclear functions. Biochem. J. 342(Pt. 2), 249-268 (1999).

2. Pascal, J. M. The comings and goings of PARP-1 in response to DNA damage. DNA Repair (Amst.) 71, 177-182 (2018).

3. Langelier, M. F., Riccio, A. A. \& Pascal, J. M. PARP-2 and PARP-3 are selectively activated by $5^{\prime}$ phosphorylated DNA breaks through an allosteric regulatory mechanism shared with PARP-1. Nucleic Acids Res. 42, 7762-7775 (2014).

4. Gibson, B. A. \& Kraus, W. L. New insights into the molecular and cellular functions of poly(ADP-ribose) and PARPs. Nat. Rev. Mol. Cell Biol. 13, 411-424 (2012).

5. Liu, C., Vyas, A., Kassab, M. A., Singh, A. K. \& Yu, X. The role of poly ADPribosylation in the first wave of DNA damage response. Nucleic Acids Res. 45, 8129-8141 (2017).
6. Martin-Hernandez, K., Rodriguez-Vargas, J. M., Schreiber, V. \& Dantzer, F. Expanding functions of ADP-ribosylation in the maintenance of genome integrity. Semin. Cell Dev. Biol. 63, 92-101 (2017).

7. Pommier, Y., O'Connor, M. J. \& de Bono, J. Laying a trap to kill cancer cells: PARP inhibitors and their mechanisms of action. Sci. Transl. Med. 8, 362ps317 (2016).

8. Murai, J. et al. Trapping of PARP1 and PARP2 by clinical PARP inhibitors. Cancer Res. 72, 5588-5599 (2012).

9. Shao, Z. et al. Clinical PARP inhibitors do not abrogate PARP1 exchange at DNA damage sites in vivo. Nucleic Acids Res. 48, 9694-9709 (2020).

10. Juhasz, S. et al. The chromatin remodeler ALC1 underlies resistance to PARP inhibitor treatment.Sci. Adv. 6, eabb8626 (2020).

11. Patel, A. G. et al. Enhanced killing of cancer cells by poly(ADP-ribose) polymerase inhibitors and topoisomerase I inhibitors reflects poisoning of both enzymes. J. Biol. Chem. 287, 4198-4210 (2012).

12. Pettitt, S. J. et al. A genetic screen using the PiggyBac transposon in haploid cells identifies Parpl as a mediator of Olaparib toxicity. PLoS ONE 8, e61520 (2013).

13. Zandarashvili, L. et al. Structural basis for allosteric PARP-1 retention on DNA breaks.Science 368, eaax6367 (2020).

14. Satoh, M. S. \& Lindahl, T. Role of poly(ADP-ribose) formation in DNA repair. Nature 356, 356-358 (1992).

15. Hopkins, T. A. et al. Mechanistic dissection of PARP1 trapping and the impact on in vivo tolerability and efficacy of PARP inhibitors. Mol. Cancer Res. 13, 1465-1477 (2015).

16. Zahradka, P. \& Ebisuzaki, K. A shuttle mechanism for DNA-protein interactions. The regulation of poly(ADP-ribose) polymerase. Eur. J. Biochem. 127, 579-585 (1982).

17. Ferro, A. M. \& Olivera, B. M. Poly(ADP-ribosylation) in vitro. Reaction parameters and enzyme mechanism. J. Biol. Chem. 257, 7808-7813 (1982).

18. Kruger, A., Burkle, A., Hauser, K. \& Mangerich, A. Real-time monitoring of PARP1-dependent PARylation by ATR-FTIR spectroscopy. Nat. Commun. 11, 2174 (2020).

19. Leidecker, O. et al. Serine is a new target residue for endogenous ADPribosylation on histones. Nat. Chem. Biol. 12, 998-1000 (2016).

20. Bonfiglio, J. J. et al. Serine ADP-ribosylation depends on HPF1. Mol. Cell 65 , 932-940.e6 (2017)

21. Larsen, S. C., Hendriks, I. A., Lyon, D., Jensen, L. J. \& Nielsen, M. L. Systemswide analysis of serine ADP-ribosylation reveals widespread occurrence and site-specific overlap with phosphorylation. Cell Rep. 24, 2493-2505.e4 (2018)

22. Hendriks, I. A., Larsen, S. C. \& Nielsen, M. L. An advanced strategy for comprehensive profiling of ADP-ribosylation sites using mass spectrometrybased proteomics. Mol. Cell Proteom. 18, 1010-1026 (2019).

23. Buch-Larsen, S. C. et al. Mapping physiological ADP-ribosylation using activated ion electron transfer dissociation. Cell Rep. 32, 108176 (2020).

24. Hendriks, I. A. et al. The regulatory landscape of the human HPF1- and ARH3-dependent ADP-ribosylome. Preprint at https://www.biorxiv.org/ content/10.1101/2021.01.26.428255v1 (2021)

25. Palazzo, L. et al. Serine is the major residue for ADP-ribosylation upon DNA damage.eLife 7, e34334 (2018).

26. Bonfiglio, J. J. et al. An HPF1/PARP1-based chemical biology strategy for exploring ADP-ribosylation. Cell 183, 1086-1102.e23 (2020) .

27. Gibbs-Seymour, I., Fontana, P., Rack, J. G. M. \& Ahel, I. HPF1/C4orf27 is a PARP-1-interacting protein that regulates PARP-1 ADP-ribosylation activity. Mol. Cell 62, 432-442 (2016).

28. Bilokapic, S., Suskiewicz, M. J., Ahel, I. \& Halic, M. Bridging of DNA breaks activates PARP2-HPF1 to modify chromatin. Nature 585, 609-613 (2020).

29. Suskiewicz, M. J. et al. HPF1 completes the PARP active site for DNA damageinduced ADP-ribosylation. Nature 579, 598-602 (2020).

30. Abplanalp, J. et al. Proteomic analyses identify ARH3 as a serine mono-ADPribosylhydrolase. Nat. Commun. 8, 2055 (2017).

31. Fontana, P. et al. Serine ADP-ribosylation reversal by the hydrolase ARH3. eLife 6, e28533 (2017).

32. Leslie Pedrioli, D.M. et al. Comprehensive ADP-ribosylome analysis identifies tyrosine as an ADP-ribose acceptor site.EMBO Rep. 19, e45310 (2018).

33. Bartlett, E. et al. Interplay of histone marks with serine ADP-ribosylation. Cell Rep. 24, 3488-3502.e5 (2018)

34. Thorsell, A. G. et al. Structural basis for potency and promiscuity in poly (ADP-ribose) polymerase (PARP) and tankyrase inhibitors. J. Med Chem. 60 , $1262-1271$ (2017)

35. Lindgren, A. E. et al. PARP inhibitor with selectivity toward ADPribosyltransferase ARTD3/PARP3. ACS Chem. Biol. 8, 1698-1703 (2013).

36. Huang, S. M. et al. Tankyrase inhibition stabilizes axin and antagonizes Wnt signalling. Nature 461, 614-620 (2009).

37. James, D. I. et al. First-in-class chemical probes against poly(ADP-ribose) glycohydrolase (PARG) inhibit DNA repair with differential pharmacology to Olaparib. ACS Chem. Biol. 11, 3179-3190 (2016). 
38. Moynahan, M. E., Chiu, J. W., Koller, B. H. \& Jasin, M. Brcal controls homology-directed DNA repair. Mol. Cell 4, 511-518 (1999).

39. Moynahan, M. E., Pierce, A. J. \& Jasin, M. BRCA2 is required for homologydirected repair of chromosomal breaks. Mol. Cell 7, 263-272 (2001).

40. Bryant, H. E. et al. Specific killing of BRCA2-deficient tumours with inhibitors of poly(ADP-ribose) polymerase. Nature 434, 913-917 (2005).

41. Farmer, H. et al. Targeting the DNA repair defect in BRCA mutant cells as a therapeutic strategy. Nature 434, 917-921 (2005).

42. Fisher, A. E., Hochegger, H., Takeda, S. \& Caldecott, K. W. Poly(ADP-ribose) polymerase 1 accelerates single-strand break repair in concert with poly(ADPribose) glycohydrolase. Mol. Cell Biol. 27, 5597-5605 (2007).

43. Noel, G. et al. Radiosensitization by the poly(ADP-ribose) polymerase inhibitor 4-amino-1,8-naphthalimide is specific of the $S$ phase of the cell cycle and involves arrest of DNA synthesis. Mol. Cancer Ther. 5, 564-574 (2006).

44. Schoonen, P. M. et al. Progression through mitosis promotes PARP inhibitorinduced cytotoxicity in homologous recombination-deficient cancer cells. Nat. Commun. 8, 15981 (2017).

45. Michelena, J. et al. Analysis of PARP inhibitor toxicity by multidimensional fluorescence microscopy reveals mechanisms of sensitivity and resistance. Nat. Commun. 9, 2678 (2018).

46. Dawicki-McKenna, J. M. et al. PARP-1 activation requires local unfolding of an autoinhibitory domain. Mol. Cell 60, 755-768 (2015).

47. Irwin, C. P. et al. PARPi-FL-a fluorescent PARP1 inhibitor for glioblastoma imaging. Neoplasia 16, 432-440 (2014).

48. Rudolph, J., Roberts, G. \& Luger, K. Histone parylation factor 1 contributes to the inhibition of PARP1 by cancer drugs. Nat. Commun. 12, 736 (2021).

49. Rudolph, J., Roberts, G., Muthurajan, U. M. \& Luger, K. HPF1 and nucleosomes mediate a dramatic switch in activity of PARP1 from polymerase to hydrolase. eLife 2021, e65773 (2021) .

50. Li, P., Zhen, Y. \& Yu, Y. Site-specific analysis of the Asp- and Glu-ADPribosylated proteome by quantitative mass spectrometry. Methods Enzymol. 626, 301-321 (2019).

51. Daniels, C. M., Ong, S. E. \& Leung, A. K. The promise of proteomics for the study of ADP-ribosylation. Mol. Cell 58, 911-924 (2015).

52. Naegeli, H., Loetscher, P. \& Althaus, F. R. Poly ADP-ribosylation of proteins. Processivity of a post-translational modification. J. Biol. Chem. 264, 14382-14385 (1989).

53. Bryant, H. E. et al. PARP is activated at stalled forks to mediate Mre11dependent replication restart and recombination. EMBO J. 28, 2601-2615 (2009).

54. DeWeirdt, P. C. et al. Genetic screens in isogenic mammalian cell lines without single cell cloning. Nat. Commun. 11, 752 (2020).

55. Hewitt, G. et al. Defective ALC1 nucleosome remodeling confers PARPi sensitization and synthetic lethality with HRD. Mol. Cell 81, 767-783.e11 (2021) .

56. Cancer Genome Atlas Research Network. Integrated genomic analyses of ovarian carcinoma. Nature 474, 609-615 (2011).

57. Oplustilova, L. et al. Evaluation of candidate biomarkers to predict cancer cell sensitivity or resistance to PARP-1 inhibitor treatment. Cell Cycle 11, 3837-3850 (2012).

58. Conrad, L. B. et al. ADP-ribosylation levels and patterns correlate with gene expression and clinical outcomes in ovarian cancers. Mol. Cancer Ther. 19, 282-291 (2020).

59. Bianchi, A. et al. PARP-1 activity (PAR) determines the sensitivity of cervical cancer to Olaparib. Gynecol. Oncol. 155, 144-150 (2019).

60. Gogola, E. et al. Selective loss of PARG restores PARylation and counteracts PARP inhibitor-mediated synthetic lethality. Cancer Cell 33, 1078-1093 (2018). e1012.

61. Slade, D. et al. The structure and catalytic mechanism of a poly(ADP-ribose) glycohydrolase. Nature 477, 616-620 (2011).

62. Stewart, G. S. et al. The RIDDLE syndrome protein mediates a ubiquitindependent signaling cascade at sites of DNA damage. Cell 136, 420-434 (2009).

63. Mehrotra, P. V. et al. DNA repair factor APLF is a histone chaperone. Mol. Cell 41, 46-55 (2011).

64. Nikolovska-Coleska, Z. et al. Development and optimization of a binding assay for the XIAP BIR3 domain using fluorescence polarization. Anal. Biochem. 332, 261-273 (2004).
65. Sellou, H. et al. The poly(ADP-ribose)-dependent chromatin remodeler Alc1 induces local chromatin relaxation upon DNA damage. Mol. Biol. Cell 27, 3791-3799 (2016).

\section{Acknowledgements}

We thank Kang Zhu and Rebecca Hughes for help with mutagenesis. U2OS Flp-In T-REx cells were generously provided by Fumiko Esashi, but originally came from Daniel Durocher's laboratory. The pSpCas9n(BB)-2A-Puro (PX462) V2.0 vector was a kind gift from Dr. Feng Zhang. Work in I.A.'s group is funded by the Wellcome Trust (grant numbers 101794 and 210634), BBSRC (BB/R007195/1), Ovarian Cancer Research Alliance (Collaborative Research Development Grant \#813369) and Cancer Research UK (C35050/A22284). Work in D.A.'s group is funded by the Cancer Research UK Career Development Fellowship (grant number 16304). M.J.S. is supported by an EMBO LongTerm Fellowship (ALTF 879-2017). Work in S.H.'s group is funded by the Institut National du Cancer (PLBIO-2019). R.S. is supported by the Fondation ARC pour la recherche sur le cancer (PDF20181208405). Work in I.G.-S.'s group is supported by a Cancer Research UK Career Development Fellowship (C62538/A24670). We thank the Microscopy-Rennes Imaging Center (BIOSIT, Université Rennes 1), member of the national infrastructure France-BioImaging supported by the French National Research Agency (ANR-10-INBS-04), for technical assistance.

\section{Author contributions}

I.A. conceived the project with inputs from all authors. E.P., F.Z., I.G.-S., K.S. and J.G. performed in vivo sensitivity and immunoblotting experiments. E.P. performed flow cytometry. R.S., S.Z. and S.H. performed live-cell imaging and analysed the imaging data T.A. and E.P. generated ARH3 OE cell line. F.Z. generated 293T PARP1 KO and U2OS PARP1-expressing Flp-In cells. M.J.S., A.P. and V.Z. performed in vitro ADPr assays. M.J.S. and A.P. performed inhibitor binding assays. D.A., M.L.N. and J.B. provided supporting data. M.J.S., I.A., F.Z. and E.P. wrote the paper with assistance from all authors.

\section{Competing interests}

The authors declare no competing interests.

\section{Additional information}

Supplementary information The online version contains supplementary material available at https://doi.org/10.1038/s41467-021-24361-9.

Correspondence and requests for materials should be addressed to M.J.S. or I.A

Peer review information Nature Communications thanks Nicola Curtin and the other, anonymous, reviewer(s) for their contribution to the peer review of this work.

Reprints and permission information is available at http://www.nature.com/reprints

Publisher's note Springer Nature remains neutral with regard to jurisdictional claims in published maps and institutional affiliations.

\begin{abstract}
Open Access This article is licensed under a Creative Commons Attribution 4.0 International License, which permits use, sharing, adaptation, distribution and reproduction in any medium or format, as long as you give appropriate credit to the original author(s) and the source, provide a link to the Creative Commons license, and indicate if changes were made. The images or other third party material in this article are included in the article's Creative Commons license, unless indicated otherwise in a credit line to the material. If material is not included in the article's Creative Commons license and your intended use is not permitted by statutory regulation or exceeds the permitted use, you will need to obtain permission directly from the copyright holder. To view a copy of this license, visit http://creativecommons.org/ licenses/by/4.0/.
\end{abstract}

(c) The Author(s) 2021 